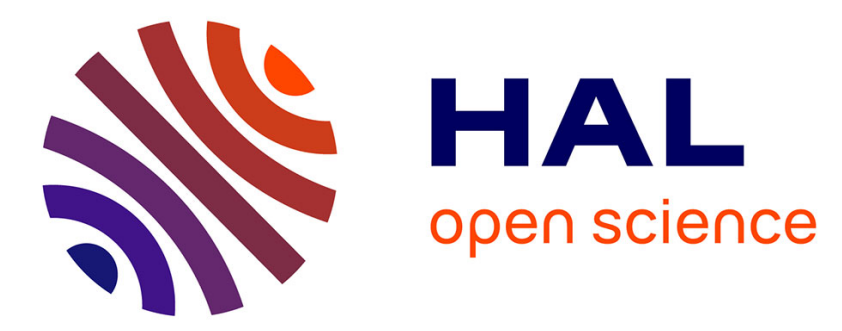

\title{
Electromagnetic inverse shape problem for coated obstacles
}

\author{
Nicolas Chaulet, Houssem Haddar
}

\section{To cite this version:}

Nicolas Chaulet, Houssem Haddar. Electromagnetic inverse shape problem for coated obstacles. Advances in Computational Mathematics, 2014, pp.21. hal-01110003

\section{HAL Id: hal-01110003 \\ https://inria.hal.science/hal-01110003}

Submitted on 27 Jan 2015

HAL is a multi-disciplinary open access archive for the deposit and dissemination of scientific research documents, whether they are published or not. The documents may come from teaching and research institutions in France or abroad, or from public or private research centers.
L'archive ouverte pluridisciplinaire HAL, est destinée au dépôt et à la diffusion de documents scientifiques de niveau recherche, publiés ou non, émanant des établissements d'enseignement et de recherche français ou étrangers, des laboratoires publics ou privés. 


\title{
Electromagnetic inverse shape problem for coated obstacles
}

\author{
N. Chaulet* $\quad$ H. Haddar ${ }^{\dagger}$
}

September 24, 2014

\begin{abstract}
We address the inverse problem of retrieving the shape of an obstacle with impedance in the form of a surface wave operator using the knowledge of electromagnetic scattering amplitude at a fixed frequency. We prove unique reconstructions from infinitely many measures. We then provide a characterization of the scattering amplitude derivative with respect to the obstacle shape. This derivative includes the case of shape dependent impedance parameters. We then employ a gradientdescent algorithm with $H^{1}$ boundary regularisation of the descent direction to numerically solve the inverse problem. The procedure is validated for three dimensional geometries using synthetic data.
\end{abstract}

Keywords. Inverse scattering problem, Maxwell's equations, Generalized Impedance Boundary Conditions, Shape derivative, Steepest descent method

\section{Introduction}

We investigate the inverse problem of retrieving the shape of coated obstacles from electromagnetic measurements of the scattering amplitude at a fixed frequency. The specificity of our work compared to the vast literature on inverse scattering problems [14], is to address coating models for which the impedance is written as a surface wave operator. They can also be referred to as generalized impedance boundary conditions $[16,4]$. They provide accurate models for thin dielectric coatings, imperfectly conducting obstacles or corrugated surfaces [17] and they can also be used as models for plasmonic waves [30]. Considering inverse problems with generalised impedance boundary conditions has been proposed in [7] and further developed in $[5,9,10,12]$.

The focus of our work is on the use of shape optimisation techniques to solve the above mentioned inverse problem. Using an adaptation of the mixed-reciprocity technique [23, 29], we first investigate the identifiability issue and prove in particular uniqueness of the shape reconstruction (independently from the operator coefficients) from the knowledge of infinitely many scattering amplitudes associated with different directions of incident waves. The case of finitely many measures is known to be challenging and left open.

We then address the main topic of this work: characterise the shape derivative of the electromagnetic scattering amplitude. This problem is technically hard, first due to the inherent complexity of Maxwell's equations and second due to the presence of a surface wave operator in the boundary condition. We employ a methodology similar to the one in $[25,18]$ where the expression of the derivative is determined using the integral representation of the solution in terms of the Green's function of the unperturbed domain. Although based on integral representations of the solutions, the method leads to an explicit expression of the derivative in terms of surface differential operators. Moreover, one is able to deal with cases where the impedance operator coefficients are also unknown and to define a derivative that depends on the geometry and the impedance operator. This type of derivative has been proposed in [5] for the scalar case and shown to be useful in simultaneous reconstructions of the geometry and the impedance coefficients.

We then exploit the derivative to solve the inverse scattering problem using a gradient descent technique associated with a least squares misfit functional. We employ an adjoint-state technique in order to compute the cost functional's derivative. The slightly uncommon feature of our algorithm is the incorporation of a surface $H^{1}$ regularisation of the descent direction by solving a surface Laplace Beltrami problem. This smoothing is stronger than the one usually used in shape optimisation problems $[1,2]$

*Department of Mathematics, University College London, Gower street, London, WC1E 6BT, UK. The research of N. C. is supported by the Medical Research Council Grant MR/K00767X/1.

${ }^{\dagger}$ INRIA Saclay Ile de France et Ecole Polytechnique, Route de Saclay, 91128 Palaiseau Cedex, France 
and turned out to be essential in order to stabilise the inversion. This methodology has the advantage of freeing the number of parameters (mesh points) used to parametrise the geometry during iterations. Indeed the expression of the shape derivative can also be used in parametrisation-dependent techniques, such as Newton type methods $[26,21,19,22]$, but this issue will not be discussed in the present work.

We conclude our paper with validating 3D numerical examples for generalised impedance boundary conditions. The problem is solved using FreeFem++ [20] and Nédélec's edge elements. The radiation condition is ensured using a first order radiation condition on a surface that encloses the computational domain. Inverse crime is avoided in our simulations since the $3 \mathrm{D}$ mesh is different at each iteration step and the final mesh (and reconstructed domain) are quite different from the one used to compute the synthetic data.

The outline of our article is as follows. We formulate the direct and inverse scattering problems in Section 2. We investigate in Section 3 the uniqueness issue for infinitely many measures after proving mixed reciprocity relations. Section 4 contains the main result of this paper related to the scattering amplitude derivative with respect to the obstacle shape. Section 5 is dedicated to the presentation of the inversion scheme based on gradient descent with regularised descent direction and an adjoint technique method to compute the cost functional derivative. We end this section with some three dimensional validating numerical results. The proofs of some technical results for surface differential operators is given in an appendix.

\section{The forward and inverse problems}

\subsection{The Generalized Impedance Boundary Condition scattering problem}

Let $\Omega$ be a bounded open set of $\mathbb{R}^{3}$ with $C^{2}$ boundary $\Gamma$. We denote by $\nu$ the outward unit normal to $\Gamma$ and by $\Omega_{\text {ext }}:=\mathbb{R}^{3} \backslash \bar{\Omega}$ that we assume to be a connected set. The scattering of an incident electromagnetic wave $\left(\mathrm{E}^{i}, \mathrm{H}^{i}\right)$ by an obstacle characterised by a Generalized Impedance Boundary Condition (GIBC) gives rise to a scattered electromagnetic wave $\left(\mathrm{E}^{s}, \mathrm{H}^{s}\right)$ that solves

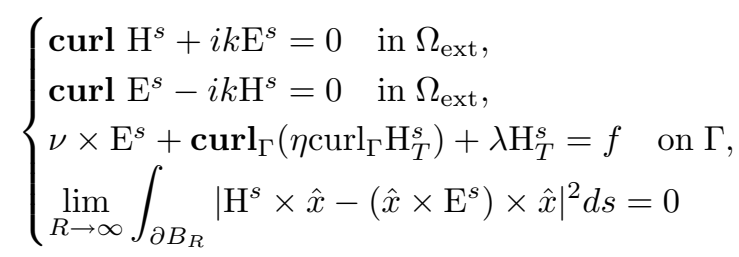

with

$$
f:=-\left(\nu \times \mathrm{E}^{i}+\operatorname{curl}_{\Gamma}\left(\eta \operatorname{curl}_{\Gamma} \mathrm{H}_{T}^{i}\right)+\lambda \mathrm{H}_{T}^{i}\right) \quad \text { on } \Gamma .
$$

In these equations, $B_{R}$ is the ball of radius $R, \hat{x}=x /|x|, k$ is the wavenumber of the incident wave and for any vector field $V \in\left(L^{2}(\Gamma)\right)^{3}$ we denote by $V_{T}:=(\nu \times V) \times \nu$ its tangential component that belongs to $L_{t}^{2}(\Gamma):=\left\{V \in\left(L^{2}(\Gamma)\right)^{3} \mid V \cdot \nu=0\right\}$. The differential operators $\operatorname{curl}_{\Gamma}$ and $\operatorname{curl}_{\Gamma}$ are respectively the scalar and vector surface curl operators which are adjoint to each other. We refer to [28, section 2.5.6] for a precise definition of these operators (see also (3) below). Finally, the parameters $\lambda$ and $\eta$ in the boundary condition are two complex valued functions of $L^{\infty}(\Gamma)$. Problem (1) is well defined for any $f \in H_{\text {curl }_{\Gamma}}(\Gamma)^{*}$ the dual space of $H_{\operatorname{curl}_{\Gamma}}(\Gamma):=\left\{V \in L_{t}^{2}(\Gamma) \mid \operatorname{curl}_{\Gamma} V \in L^{2}(\Gamma)\right\}$ which is endowed with the graph norm. Let us introduce the spaces

$$
\begin{aligned}
& H_{\text {curl }}^{\text {ext }}\left(\Omega_{\text {ext }}\right):=\left\{V \in\left(\mathcal{D}^{\prime}\left(\Omega_{\text {ext }}\right)\right)^{3} \mid \varphi V \in H_{\text {curl }}\left(\Omega_{\text {ext }}\right), \forall \varphi \in C_{0}^{\infty}\left(\mathbb{R}^{3}\right\}\right. \\
& V_{\mathrm{H}}\left(\Omega_{\text {ext }}\right):=\left\{V \in H_{\text {curl }}^{\text {ext }}\left(\Omega_{\text {ext }}\right) \mid V_{T} \in H_{\text {curl }_{\Gamma}}(\Gamma)\right\}
\end{aligned}
$$

where for any open set $D, H_{\text {curl }}(D):=\left\{V \in\left(L^{2}(D)\right)^{3} \mid \operatorname{curl} V \in\left(L^{2}(D)\right)^{3}\right\}$. We also need the Hilbert space

$$
V_{H, R}:=\left\{V \in H_{\text {curl }}\left(\Omega \cap B_{R}\right) \mid V_{T} \in H_{\operatorname{curl}_{\Gamma}}(\Gamma)\right\} .
$$

endowed with the graph norm denoted by $\|\cdot\|_{V_{H, R}}$. Let us recall the following theorem from [11].

Theorem 2.1. Let $(\lambda, \eta) \in\left(L^{\infty}(\Gamma)\right)^{2}$ be such that

$$
\Re(\lambda) \geq 0, \quad \Re(\eta) \geq 0, \quad|\lambda| \geq c \text { and }|\eta| \geq c \quad \text { a.e. on } \Gamma
$$


for some constant $c>0$, and assume the imaginary parts of $\lambda$ and $\eta$ do not change sign on $\Gamma$. Then for all $f \in H_{\text {curl }_{\Gamma}}(\Gamma)^{*}$ problem (1) has a unique solution $\left(\mathrm{E}^{s}, \mathrm{H}^{s}\right) \in H_{\text {curl }}^{\text {ext }}\left(\Omega_{\mathrm{ext}}\right) \times V_{\mathrm{H}}\left(\Omega_{\mathrm{ext}}\right)$. Moreover, for all ball $B_{R}$ that contains $\bar{\Omega}$ there exists $C_{R}>0$ independent of $f$ such that

$$
\left\|\mathrm{E}^{s}\right\|_{H_{\mathrm{curl}}\left(\Omega_{R}\right)}+\left\|\mathrm{H}^{s}\right\|_{V_{H, R}} \leq C_{R}\|f\|_{H_{\mathrm{curl}_{\Gamma}(\Gamma) *}}
$$

where $\Omega_{R}:=\Omega \cap B_{R}$.

From now on, we assume that the impedance functions $\lambda$ and $\eta$ satisfy the assumptions of Theorem 2.1. In the following we will need additional regularity for the solution to problem 1 . In this view, let us introduce the surface divergence operator $\operatorname{div}_{\Gamma}$ which is the negative adjoint of the surface gradient $\nabla_{\Gamma}$. We recall the following algebraic relations that link the different differential operators introduced here above

$$
\left.\nu \cdot(\operatorname{curl} V)\right|_{\Gamma}=\operatorname{curl}_{\Gamma} V_{T}=-\operatorname{div}_{\Gamma}(\nu \times V) \text { and } \operatorname{curl}_{\Gamma} v=-\nu \times \nabla_{\Gamma} v
$$

where $V$ is vector field defined in a neighbourhood of $\Gamma$ and $v$ is a function defined on $\Gamma$. For regular boundaries $\left(C^{\infty}\right)$ we also define fractional Sobolev spaces $H_{\operatorname{div}_{\Gamma}}^{s}(\Gamma):=\left\{V \in H_{t}^{s}(\Gamma) \mid \operatorname{div}_{\Gamma} V \in H^{s}(\Gamma)\right\}$ and $H_{\operatorname{curl}_{\Gamma}}^{s}(\Gamma):=\left\{V \in H_{t}^{s}(\Gamma) \mid \operatorname{curl}_{\Gamma} V \in H^{s}(\Gamma)\right\}$ where $H_{t}^{s}(\Gamma)$ is the closure of $\left\{V \in\left(C^{\infty}(\Gamma)\right)^{3} \mid V \cdot \nu=0\right\}$ in $\left(H^{s}(\Gamma)\right)^{3}$ and $s \in \mathbb{R}$.

Under additional regularity assumptions on $\lambda, \eta$ and $\Gamma$ we have the following regularity property for the electromagnetic field.

Proposition 2.2. Let $\Gamma$ be of class $C^{s+2}$, and let us assume that $\lambda$ and $\eta$ are in $C^{s+1}(\Gamma)$. For any $f \in H_{\operatorname{curl}_{\Gamma}}(\Gamma)^{*} \cap H_{\operatorname{div}_{\Gamma}}^{s-1 / 2}(\Gamma)$ for $s \geq 0$, if $(\mathrm{E}, \mathrm{H}) \in H_{\text {curl }}^{\text {ext }}\left(\Omega_{\mathrm{ext}}\right) \times V_{\mathrm{H}}\left(\Omega_{\mathrm{ext}}\right)$ satisfies problem (1) then $(\mathrm{E}, \mathrm{H}) \in\left(H^{s+1}\left(\Omega_{R}\right)^{3} \times\left(H^{s+1}\left(\Omega_{R}\right)\right)^{3}\right.$ for all ball $B_{R}$ of radius $R$ such that $\bar{\Omega} \subset B_{R}$.

Proof. Using $\operatorname{div}_{\Gamma} \operatorname{curl}_{\Gamma}=0$, the boundary condition satisfied by $(\mathrm{E}, \mathrm{H}) \operatorname{implies}_{\operatorname{div}_{\Gamma}}\left(\lambda \mathrm{H}_{T}\right)=-\operatorname{div}_{\Gamma}(\nu \times$ $\mathrm{E})+\operatorname{div}_{\Gamma} f \in H^{-1 / 2}(\Gamma)$ since $\mathrm{E} \in H_{\text {curl }}^{\text {ext }}\left(\Omega_{\text {ext }}\right)$ and $f$ is in $H_{\operatorname{div}_{\Gamma}}^{-1 / 2}(\Gamma)$. Combined with $\operatorname{curl}_{\Gamma} \mathrm{H}_{T} \in L^{2}(\Gamma)$ because $\mathrm{H}_{T} \in H_{\operatorname{curl}_{\Gamma}}(\Gamma)$, this implies $\mathrm{H}_{T} \in\left(H^{1 / 2}(\Gamma)\right)^{3}$. Hence, applying classical regularity results for Maxwell's equations [3, Corollary 2.15] to the magnetic field $\mathrm{H}$, we obtain $\mathrm{H} \in\left(H^{1}\left(\Omega_{R}\right)\right)^{3}$. In addition, $\operatorname{curl}_{\Gamma}\left(\eta \operatorname{curl}_{\Gamma} \mathrm{H}_{T}\right)=-\nu \times \mathrm{E}-\lambda \mathrm{H}_{T}+f \in H^{-1 / 2}(\Gamma)$ whence $\operatorname{curl}_{\Gamma} \mathrm{H}_{T} \in H^{1 / 2}(\Gamma)$ and from Maxwell's equations we have

$$
\nu \cdot \mathrm{E}=-\frac{1}{i k} \nu \cdot \operatorname{curl} \mathrm{H}=-\frac{1}{i k} \operatorname{curl}_{\Gamma} \mathrm{H}_{T} \in H^{1 / 2}(\Gamma) .
$$

Therefore, using again regularity results [3, Corollary 2.15], we deduce that $\mathrm{E} \in\left(H^{1}\left(\Omega_{R}\right)\right)^{3}$. To obtain further regularity let us remark that similarly to equation (4) we have

$$
\operatorname{div}_{\Gamma}(\nu \times \mathrm{E})=-\nu \cdot \operatorname{curl} \mathrm{E}=-i k \nu \cdot \mathrm{H} \in H^{1 / 2}(\Gamma) .
$$

We then obtain the desired result by induction on $s$.

\subsection{Statement of the inverse problem}

We recall that any solution $(\mathrm{E}, \mathrm{H})$ to Maxwell's equations

$$
\text { curl } \mathrm{H}+i k \mathrm{E}=0, \quad \operatorname{curl} \mathrm{E}-i k \mathrm{H}=0
$$

outside some bounded Lipschitz domain $D$ that also satisfies the Silver-Müller radiation condition (the last equation in system (1)) admits the following asymptotic behaviour

$$
\mathrm{E}(x)=\frac{e^{i k|x|}}{|x|}\left(\mathrm{E}^{\infty}(\hat{x})+\mathcal{O}\left(\frac{1}{|x|}\right)\right), \quad \mathrm{H}(x)=\frac{e^{i k|x|}}{|x|}\left(\mathrm{H}^{\infty}(\hat{x})+\mathcal{O}\left(\frac{1}{|x|}\right)\right) \quad|x| \rightarrow+\infty
$$

uniformly for all direction $\hat{x} \in S^{2}$ where $S^{2}$ denotes the unit sphere of $\mathbb{R}^{3}$. This asymptotic behaviour uniquely defines the far field patterns $\mathrm{E}^{\infty}$ and $\mathrm{H}^{\infty}$ as functions of $L_{t}^{2}\left(S^{2}\right)$ and we have the following representation formula

$$
\mathrm{E}^{\infty}(\hat{x})=\frac{i k}{4 \pi} \hat{x} \times \int_{\partial D}\{\nu(y) \times \mathrm{E}(y)+[\nu(y) \times \mathrm{H}(y)] \times \hat{x}\} e^{-i k \hat{x} \cdot y} d s(y),
$$

and $\mathrm{H}^{\infty}(\hat{x})=\hat{x} \times \mathrm{E}^{\infty}(\hat{x})$. We refer to [14] or [24] for general results about electromagnetic scattering theory. 
Following the notations in [24] let us introduce the incident electromagnetic plane waves with incidence direction $\hat{\theta} \in S^{2}$ as being described by the matrices $\mathscr{E}^{i}(x, \hat{\theta})$ and $\mathscr{H}^{i}(x, \hat{\theta})$ which are defined for a polarisation $\mathbf{p} \in S^{2}$ by

$$
\begin{aligned}
\mathscr{E}^{i}(x, \hat{\theta}) \mathbf{p} & :=-\frac{1}{i k} \operatorname{curl}_{x} \operatorname{curl}_{x}\left(\mathbf{p} e^{i k x \cdot \hat{\theta}}\right)=i k((\hat{\theta} \times \mathbf{p}) \times \hat{\theta}) e^{i k x \cdot \hat{\theta}} \\
\mathscr{H}^{i}(x, \hat{\theta}) \mathbf{p} & :=\operatorname{curl}_{x}\left(\mathbf{p} e^{i k x \cdot \hat{\theta}}\right)=i k(\hat{\theta} \times \mathbf{p}) e^{i k x \cdot \hat{\theta}}
\end{aligned}
$$

Each pair of corresponding columns of $\mathscr{E}^{i}(\cdot, \hat{\theta})$ and $\mathscr{H}^{i}(\cdot, \hat{\theta})$ satisfy Maxwell's equations $(5)$ in $\mathbb{R}^{3}$. Since problem (1) is linear with respect to the right-hand side $f$, we can define the scattered field matrices $\mathscr{E}^{s}(\cdot, \hat{\theta})$ and $\mathscr{H}^{s}(\cdot, \hat{\theta})$ where for any polarisation $\mathbf{p} \in S^{2}$, the electromagnetic field $\left(\mathscr{E}^{s}(x, \hat{\theta}) \mathbf{p}, \mathscr{H}^{s}(x, \hat{\theta}) \mathbf{p}\right)$ solves problem (1) with $f$ being given by $(2)$ for $\mathrm{E}^{i}(x)=\mathscr{E}^{i}(x, \hat{\theta}) \mathbf{p}$ and $\mathrm{H}^{i}(x)=\mathscr{H}^{i}(x, \hat{\theta}) \mathbf{p}$. We also denote by $\mathscr{E}^{\infty}(\hat{x}, \hat{\theta})$ the matrix representation of the far field pattern associated with $\mathscr{E} s(x, \hat{\theta})$ and by

$$
\mathscr{E}(x, \hat{\theta})=\mathscr{E}^{i}(x, \hat{\theta})+\mathscr{E}^{s}(x, \hat{\theta}) \quad \text { and } \quad \mathscr{H}(x, \hat{\theta})=\mathscr{H}^{i}(x, \hat{\theta})+\mathscr{H}^{s}(x, \hat{\theta})
$$

the total fields. In what follows, we will frequently make use this matrix field notation. We define the curl of a matrix $M=\left(m_{1}, m_{2}, m_{3}\right)$ where $m_{1}, m_{2}$ and $m_{3}$ are column vectors as being given by the matrix $\operatorname{curl} M:=\left(\operatorname{curl} m_{1}, \operatorname{curl} m_{2}, \operatorname{curl} m_{3}\right)$. Similarly, for $M=\left(m_{1}, m_{2}, m_{3}\right)^{\mathrm{T}}$ where $m_{1}, m_{2}$ and $m_{3}$ are row vectors we define the divergence of $M$ as the column vector $\operatorname{div} M:=\left(\operatorname{div}\left(m_{1}\right), \operatorname{div}\left(m_{2}\right), \operatorname{div}\left(m_{3}\right)\right)^{\mathrm{T}}$ where $\mathrm{T}^{\mathrm{T}}$ stands for the transpose of a vector or a matrix. Moreover, the surface differential operator $\operatorname{curl}_{\Gamma}$ is defined for $M$ by the row vector field $\operatorname{curl}_{\Gamma} M:=\left(\operatorname{curl}_{\Gamma} m_{1}, \operatorname{curl}_{\Gamma} m_{2}, \operatorname{curl}_{\Gamma} m_{3}\right)$ and the vector $\operatorname{curl}_{\Gamma}$ operator is defined for a row vector field $V=\left(v_{1}, v_{2}, v_{3}\right)$ by the matrix $\operatorname{curl}_{\Gamma} V:=\left(\operatorname{curl}_{\Gamma} v_{1}, \operatorname{curl}_{\Gamma} v_{2}, \operatorname{curl}_{\Gamma} v_{3}\right)$. Finally, for a column vector $V$ and a matrix $M$ we set $V \times M=-M \times V:=\left(V \times m_{1}, V \times m_{2}, V \times m_{3}\right)$ and by extension $M_{T}:=(\nu \times M) \times \nu$ where the normal vector $\nu$ is considered as a column vector.

For fixed $\lambda$ and $\eta$ we address the question of reconstructing the shape $\Gamma$ from the knowledge of the far field pattern $\mathrm{E}^{\infty}(\hat{x}, \hat{\theta})$ for any $(\hat{x}, \hat{\theta})$. We establish in the next section a uniqueness result for this inverse problem and in section 4 we compute the derivatives of the far field patterns with respect to $\Gamma$. This derivative can then be used to solve the inverse problem with a non linear optimisation technique as the one presented in section 5. We shall numerically demonstrate that in practice a small number of incident waves would be sufficient to obtain accurate shape reconstructions.

\section{Uniqueness for infinitely many incident waves}

We prove in this section that the knowledge of $\mathscr{E} \infty(\hat{x}, \hat{\theta})$ for all $(\hat{x}, \hat{\theta}) \in\left(S^{2}\right)^{3}$ uniquely determines $\Gamma$. The main result of this section is stated in the theorem below and can be seen as an extension of the case $\eta=0$ treated in [8, Theorem 3.1] for example.

Theorem 3.1. Let $\Gamma_{1}$ and $\Gamma_{2}$ be two boundaries of class $C^{2}$ and $\lambda$ and $\eta$ be two complex valued functions defined on $\Gamma_{1}$ and $\Gamma_{2}$ such that $\lambda \in C^{1}\left(\Gamma_{1}\right) \cap C^{1}\left(\Gamma_{2}\right)$ and $\eta \in C^{1}\left(\Gamma_{1}\right) \cap C^{1}\left(\Gamma_{2}\right)$ satisfy the assumptions of Theorem 2.1. Let us denote by $\mathscr{E}_{1}^{\infty}$ (respectively $\mathscr{E}_{2}^{\infty}$ ) the far field pattern associated with $\Gamma_{1}, \lambda$ and $\eta$ (respectively $\Gamma_{2}, \lambda$ and $\left.\eta\right)$. If $\mathscr{E}_{1}^{\infty}(\hat{x}, \hat{\theta})=\mathscr{E}_{2}^{\infty}(\hat{x}, \hat{\theta})$ for all $\hat{x}, \hat{\theta} \in S^{2}$ then $\Gamma_{1}=\Gamma_{2}$.

To prove this result we need first to establish a mixed reciprocity relation (see Lemma 3.2) that has been first obtained by Potthast in [29]. This result is the corner stone of the proof of uniqueness for $\Gamma$ which does not depend on the values of $\lambda$ and $\eta$. Let us first introduce the electromagnetic dipole located at point $z \in \Omega_{\text {ext }}$ that we represent with the matrices $\mathcal{E}^{i}(\cdot, z)$ and $\mathcal{H}^{i}(\cdot, z)$ defined for $\mathbf{p} \in S^{2}$ by

$$
\begin{gathered}
\mathcal{E}^{i}(x, z) \mathbf{p}:=-\frac{1}{i k} \operatorname{curl}_{x} \operatorname{curl}_{x}(\mathbf{p} \Phi(x, z)), \\
\mathcal{H}^{i}(x, z) \mathbf{p}:=\operatorname{curl}_{x}(\mathbf{p} \Phi(x, z))=\nabla_{x}(\Phi(x, z)) \times \mathbf{p}
\end{gathered}
$$

where

$$
\Phi(x, z)=\frac{1}{4 \pi} \frac{e^{i k|x-z|}}{|x-z|} \text { for } x \neq z
$$

is the outgoing Green's function for the Helmholtz' equation in $\mathbb{R}^{3}$. By linearity, we define the scattered field matrices $\mathcal{E}^{s}(\cdot, z)$ and $\mathcal{H}^{s}(\cdot, z)$ such that $\left(\mathcal{E}^{s}(\cdot, z) \mathbf{p}, \mathcal{H}^{s}(\cdot, z) \mathbf{p}\right)$ solves problem (1) with $f$ being given by $(2)$ for $\mathrm{E}^{i}=\mathcal{E}^{i}(\cdot, z) \mathbf{p}$ and $\mathrm{H}^{i}=\mathcal{H}^{i}(\cdot, z) \mathbf{p}$. We denote by $\mathcal{E}^{\infty}\left((\cdot, z)\right.$ and $\mathcal{H}^{\infty}(\cdot, z)$ the associated matrix far field patterns and by $\mathcal{E}:=\mathcal{E}^{i}+\mathcal{E}^{s}$ and $\mathcal{H}=\mathcal{H}^{i}+\mathcal{H}^{s}$ the matrix total fields. With this notation, the following mixed reciprocity relation holds. 
Lemma 3.2. For all $x \in \Omega_{\mathrm{ext}}$ and $\hat{\theta} \in S^{2}$ we have

$$
\left[\mathscr{E}^{s}(x,-\hat{\theta})\right]^{\mathrm{T}}=4 \pi \mathcal{E}^{\infty}(\hat{\theta}, x) .
$$

Proof. For (E, H) solution to Maxwell's equations outside $\Omega$ that satisfy the Silver-Müller radiation condition, the Stratton-Chu formula (see e.g. [24, Corollary 4.1.2.3]) writes as

$$
\mathrm{E}(x)=\int_{\Gamma}\left\{\left[\mathcal{E}^{i}(z, x)\right]^{\mathrm{T}}[\nu \times \mathrm{H}(z)]+\left[\mathcal{H}^{i}(z, x)\right]^{\mathrm{T}}[\nu \times \mathrm{E}(z)]\right\} d s(z) \quad \text { for } x \in \Omega_{\mathrm{ext}} .
$$

The associated far field pattern is given by (see e.g. [24, Corollary 4.1.3.1])

$$
4 \pi \mathrm{E}^{\infty}(\hat{\theta})=\int_{\Gamma}\left\{\left[\mathscr{H}^{i}(z,-\hat{\theta})\right]^{\mathrm{T}}[\nu \times \mathrm{E}(z)]+\left[\mathscr{E}^{i}(z,-\hat{\theta})\right]^{\mathrm{T}}[\nu \times \mathrm{H}(z)]\right\} d s(z) \quad \text { for } \hat{\theta} \in S^{2} .
$$

Moreover, for $\left(\mathrm{E}_{1}^{i}, \mathrm{H}_{1}^{i}\right)$ and $\left(\mathrm{E}_{2}^{i}, \mathrm{H}_{2}^{i}\right)$ solutions to Maxwell's equations inside $\Omega$,

$$
\int_{\Gamma}\left\{\mathrm{E}_{1}^{i} \cdot\left[\nu \times \mathrm{H}_{2}^{i}\right]+\mathrm{H}_{1}^{i} \cdot\left[\nu \times \mathrm{E}_{2}^{i}\right]\right\} d s=0
$$

and the same applies for solutions $\left(\mathrm{E}_{1}^{s}, \mathrm{H}_{1}^{s}\right)$ and $\left(\mathrm{E}_{2}^{s}, \mathrm{H}_{2}^{s}\right)$ to Maxwell's equations outside $\Omega$ that satisfy the Silver-Müller radiation condition:

$$
\int_{\Gamma}\left\{\mathrm{E}_{1}^{s} \cdot\left[\nu \times \mathrm{H}_{2}^{s}\right]+\mathrm{H}_{1}^{s} \cdot\left[\nu \times \mathrm{E}_{2}^{s}\right]\right\} d s=0
$$

Formulas (7) and (8) applied to the expressions of $\mathscr{E}^{s}(z,-\hat{\theta})$ and $\mathcal{E}^{\infty}(\hat{\theta}, z)$ imply that

$$
\begin{aligned}
{\left[\mathscr{E}^{s}(x,-\hat{\theta})\right]^{\mathrm{T}}-4 \pi \mathcal{E}^{\infty}(\hat{\theta}, x)=} & \int_{\Gamma}\left\{\left[\mathscr{H}^{s}(z,-\hat{\theta})\right]^{\mathrm{T}}\left[\mathcal{E}^{i}(z, x) \times \nu\right]+\left[\mathscr{E}^{s}(z,-\hat{\theta})\right]^{\mathrm{T}}\left[\mathcal{H}^{i}(z, x) \times \nu\right]\right. \\
& \left.+\left[\mathscr{H}^{i}(z,-\hat{\theta})\right]^{\mathrm{T}}\left[\mathcal{E}^{s}(z, x) \times \nu\right]+\left[\mathscr{E}^{i}(z,-\hat{\theta})\right]^{\mathrm{T}}\left[\mathcal{H}^{s}(z, x) \times \nu\right]\right\} d s(z)
\end{aligned}
$$

for all $x \in \Omega_{\text {ext }}$ and $\hat{\theta} \in S^{2}$. By using identities (9) and (10) we then obtain

$$
[\mathscr{E} s(x,-\hat{\theta})]^{\mathrm{T}}-4 \pi \mathcal{E}^{\infty}(\hat{\theta}, x)=\int_{\Gamma}\left\{[\mathscr{H}(z,-\hat{\theta})]^{\mathrm{T}}[\mathcal{E}(z, x) \times \nu]+[\mathscr{E}(z,-\hat{\theta})]^{\mathrm{T}}[\mathcal{H}(z, x) \times \nu]\right\} d s(z)
$$

Since the columns of $(\mathscr{E}(\cdot, \hat{\theta}), \mathscr{H}(\cdot, \hat{\theta}))$ and $(\mathcal{E}(\cdot, x), \mathcal{H}(\cdot, x))$ satisfy

$$
\nu \times \mathrm{E}=-\left\{\operatorname{curl}_{\Gamma}\left(\eta \operatorname{curl}_{\Gamma} \mathrm{H}_{T}\right)+\lambda \mathrm{H}_{T}\right\} \quad \text { on } \Gamma,
$$

the previous identity and the symmetry of the operator $\operatorname{curl}_{\Gamma}\left(\eta \operatorname{curl} \Gamma_{\Gamma}\right)+\lambda \cdot$ imply that

$$
\left[\mathscr{E}^{s}(x,-\hat{\theta})\right]^{\mathrm{T}}-4 \pi \mathcal{E}^{\infty}(\hat{\theta}, x)=0 \quad \text { for all }(x, \hat{\theta}) \in \Omega_{\mathrm{ext}} \times S^{2} .
$$

We have now the necessary tools to prove Theorem 3.1 .

Proof of Theorem 3.1. Let us assume that $\Omega_{1} \neq \Omega_{2}$ and let us define $\widetilde{\Omega}=\mathbb{R}^{3} \backslash \overline{\Omega_{1} \cup \Omega_{2}}$. From Rellich's Lemma and the unique continuation principle we have

$$
\mathscr{E}_{1}^{s}(y, \hat{\theta})=\mathscr{E}_{2}^{s}(y, \hat{\theta}) \quad \text { for all }(y, \hat{\theta}) \in \widetilde{\Omega} \times S^{2} .
$$

The mixed reciprocity principle (Lemma 3.2) then implies

$$
\mathcal{E}_{1}^{\infty}(-\hat{\theta}, y)=\mathcal{E}_{2}^{\infty}(-\hat{\theta}, y) \text { for all }(y, \hat{\theta}) \in \widetilde{\Omega} \times S^{2} .
$$

Applying once more Rellich's Lemma and the unique continuation principle we deduce from the last identity that

$$
\mathcal{E}_{1}^{s}(x, y)=\mathcal{E}_{2}^{s}(x, y) \quad \text { for all }(x, y) \in(\widetilde{\Omega})^{2} .
$$

We now make use of the singular behaviour of the dipole functions $\mathcal{E}^{i}$ and $\mathcal{H}^{i}$ to complete the proof. Let us assume that $\Omega_{1} \not \subset \Omega_{2}$. Then there exists $x_{*} \in\left(\Gamma_{1} \cap \partial \tilde{\Omega}\right) \backslash \overline{\Omega_{2}}$ and $r_{*}>0$ such that $B\left(x_{*}, r_{*}\right) \subset \mathbb{R}^{3} \backslash \overline{\Omega_{2}}$. Let 
us define the sequence of points $z_{n}:=x_{*}+\nu / n$ which approaches $x_{*}$ from outside $\widetilde{\Omega}$ (at least for large $n$ ). For $n$ sufficiently large we deduce from $(11)$ and the boundary condition satisfied by $\left(\mathcal{E}_{1}^{s}\left(\cdot, z_{n}\right), \mathcal{H}_{1}^{s}\left(\cdot, z_{n}\right)\right)$ on $\Gamma_{1}$ that

$$
\begin{aligned}
\nu \times \mathcal{E}_{2}^{s}\left(\cdot, z_{n}\right)+\operatorname{curl}_{\Gamma}\left(\eta \operatorname{curl}_{\Gamma} \mathcal{H}_{2, T}^{s}\right)\left(\cdot, z_{n}\right)+\lambda \mathcal{H}_{2, T}^{s}\left(\cdot, z_{n}\right) \\
=\nu \times \mathcal{E}_{1}^{s}\left(\cdot, z_{n}\right)+\operatorname{curl}_{\Gamma}\left(\eta \operatorname{curl}_{\Gamma} \mathcal{H}_{1, T}^{s}\right)\left(\cdot, z_{n}\right)+\lambda \mathcal{H}_{1, T}^{s}\left(\cdot, z_{n}\right) \\
=-\left\{\nu \times \mathcal{E}^{i}\left(\cdot, z_{n}\right)+\operatorname{curl}_{\Gamma}\left(\eta \operatorname{curl}_{\Gamma} \mathcal{H}_{T}^{i}\right)\left(\cdot, z_{n}\right)+\lambda \mathcal{H}_{T}^{i}\left(\cdot, z_{n}\right)\right\}
\end{aligned}
$$

on $\Gamma_{1} \cap B\left(X_{*}, r_{*}\right)$. Let us denote $P[E, H]:=\nu \times E+\operatorname{curl}_{\Gamma}\left(\eta \operatorname{curl}_{\Gamma} H_{T}\right)+\lambda H_{T}$. Since the scattering problem associated with $\Gamma_{2}$ is well posed and since $\eta \in C^{1}\left(\Gamma_{1}\right)$ and $\lambda \in C^{1}\left(\Gamma_{1}\right)$, the sequence $P\left[\mathcal{E}_{2}^{s}\left(\cdot, z_{n}\right), \mathcal{H}_{2}^{s}\left(\cdot, z_{n}\right)\right]$ converges in $\left(L^{2}\left(\Gamma_{1} \cap B\left(x_{*}, r_{*}\right)\right)\right)^{3 \times 3}$ towards $P\left[\mathcal{E}_{2}^{s}\left(\cdot, x_{*}\right), \mathcal{H}_{2}^{s}\left(\cdot, x_{*}\right)\right]$ as $n \rightarrow \infty$. Moreover, for $x_{1} \in$ $\Gamma_{1} \cap B\left(X_{*}, r_{*}\right) \backslash x_{*}$ we have

$$
\lim _{n \rightarrow \infty} P\left[\mathcal{E}^{i}\left(x_{1}, z_{n}\right), \mathcal{H}^{i}\left(x_{1}, z_{n}\right)\right]=P\left[\mathcal{E}^{i}\left(x_{1}, x_{*}\right), \mathcal{H}^{i}\left(x_{1}, x_{*}\right)\right]
$$

Therefore, by (12) we deduce that

$$
P\left[\mathcal{E}^{i}\left(\cdot, x_{*}\right), \mathcal{H}^{i}\left(\cdot, x_{*}\right)\right] \in\left(L^{2}\left(\Gamma_{1} \cap B\left(x_{*}, r_{*}\right)\right)\right)^{3 \times 3} .
$$

Similarly we obtain that $\operatorname{div}_{\Gamma}\left[P\left[\mathcal{E}^{i}\left(\cdot, x_{*}\right), \mathcal{H}^{i}\left(\cdot, x_{*}\right)\right]\right] \in\left(L^{2}\left(\Gamma_{1} \cap B\left(x_{*}, r_{*}\right)\right)\right)^{3}$ and since $\Gamma_{1}$ is of class $C^{2}$, by using Proposition 2.2 we deduce that $\mathcal{E}^{i}\left(\cdot, x_{*}\right) \in\left(H^{1}\left(\mathbb{R}^{3} \backslash \overline{\Omega_{1}} \cap B\left(x_{*}, r_{*}\right)\right)\right)^{3}$. This is in contradiction with the fact that $\mathcal{E}^{i}\left(\cdot, x_{*}\right)$ is singular at point $x_{*}$. Therefore $\Omega_{1} \subset \Omega_{2}$. The reverse inclusion also holds by symmetry and hence $\Gamma_{1}=\Gamma_{2}$.

Remark 3.3. The uniqueness result stated in Theorem 3.1 only rely on the symmetry of the impedance operator $\operatorname{curl}_{\Gamma} \eta \operatorname{curl}_{\Gamma}+\lambda$ and therefore can be extended to boundary conditions of the form

$$
\nu \times \mathrm{E}+\mathcal{Z} \mathrm{H}_{T}=0 \quad \text { on } \Gamma
$$

where $\mathcal{Z}: V(\Gamma) \rightarrow V(\Gamma)^{*}$ is a linear, bounded and symmetric operator from an Hilbert space $V(\Gamma) \subset$ $H_{\text {curl }_{\Gamma}}^{-1 / 2}(\Gamma)$ into its dual space $V(\Gamma)^{*}$. The operator $\mathcal{Z}$ has also to satisfy sign conditions which ensure that the scattering problem is well-posed (see [11]).

Remark 3.4. For a known shape $\Gamma$ of class $C^{2}$ one can establish uniqueness for the impedance functions $\lambda$ and $\eta$. To be more precise, let us denote $\Lambda$ a subset of $C^{0}(\Gamma) \cap C^{2}(\Gamma)$ that is such that any $(\lambda, \eta) \in \Lambda$ satisfy assumptions of Theorem 2.1. Then the map

$$
\Lambda \quad \longrightarrow \quad\left(L_{t}^{2}\left(S^{2} \times S^{2}\right)\right)^{3} ; \quad(\lambda, \eta) \quad \longmapsto \quad \mathscr{E}^{\infty}(\hat{x}, \hat{\theta})
$$

is injective. The proof relies on a density result for the total magnetic field stipulating that that if $f \in H_{\operatorname{curl}_{\Gamma}}(\Gamma)^{*}$ satisfies

$$
\int_{\Gamma} \mathscr{H}(x, \hat{\theta})^{\mathrm{T}} f(x) d s=0 \quad \text { for all } \hat{\theta} \in S^{2}
$$

then $f=0$ (see [12, section 6.2] for further details). The question of identifiability for a finite number of incident waves is still open. Even if one assumes that the boundary is known numerical simulations suggest that a single incident wave is not sufficient to uniquely determine $\lambda$ and $\eta$. See [7] for a discussion of the scalar case.

\section{Shape derivative of the far field pattern}

For a given direction of incidence $\hat{\theta} \in S^{2}$ and a given polarisation $\mathbf{p} \in S^{2}$ let us define the non linear functional

$$
T:(\lambda, \eta, \Gamma) \longrightarrow \mathscr{E}^{\infty}(\cdot, \hat{\theta}) \mathbf{p}
$$

where $\mathscr{E}^{\infty}(\cdot, \hat{\theta}) \mathbf{p} \in L_{t}^{2}\left(S^{2}\right)$ is the far field pattern of the solution to problem (1) with $f$ given by $(2)$ and $\left(\mathrm{E}^{i}, \mathrm{H}^{i}\right)$ given by $(6)$. In the following we assume that $\hat{\theta}$ and $\mathbf{p}$ are fixed and we do not mention explicitly the dependence of $T$ on $\hat{\theta}$ and $\mathbf{p}$. We begin this section by giving an explicit characterization of the Fréchet derivative of $T$ with respect to both, the shape in Theorem 4.1, and the impedance coefficient in Theorem 4.3. We then pursue in section 4.2 with the proof of Theorem 4.1 and we conclude in section 4.3 with a numerical validation of the result obtained in Theorem 4.1. 


\subsection{Characterization of the partial derivatives of the far field pattern}

Before giving the derivative of $T$ with respect to $\Gamma$, let us introduce some notations and give a precise definition of the shape derivative of the far-field pattern. Let $B_{r}^{\infty}$ be the open ball of $\left(C^{1, \infty}\left(\mathbb{R}^{3}\right)\right)^{3}$ of radius $r$ where $C^{1, \infty}\left(\mathbb{R}^{3}\right):=C^{1}\left(\mathbb{R}^{3}\right) \cap W^{1, \infty}\left(\mathbb{R}^{3}\right)$ and $\left(C^{1, \infty}\left(\mathbb{R}^{3}\right)\right)^{3}$ is endowed with the norm

$$
\|V\|:=\|V\|_{\left(L^{\infty}\left(\mathbb{R}^{3}\right)\right)^{3}}+\|\nabla V\|_{\left(L^{\infty}\left(\mathbb{R}^{3}\right)\right)^{3 \times 3}} .
$$

For any $\varepsilon \in B_{1}^{\infty}$ we denote $f_{\varepsilon}:=\operatorname{Id}+\varepsilon$ and $\lambda_{\varepsilon}:=\lambda \circ f_{\varepsilon}^{-1}, \eta_{\varepsilon}:=\eta \circ f_{\varepsilon}^{-1}$. We note that since $\|\varepsilon\|<1$, the map $f_{\varepsilon}$ is a $C^{1}$ diffeomorphism from $\mathbb{R}^{3}$ into $\mathbb{R}^{3}$. This map is used to define a perturbed geometry $\Omega_{\varepsilon}:=f_{\varepsilon}(\Omega)$ with boundary $\Gamma_{\varepsilon}:=f_{\varepsilon}(\Gamma)$.

For $r<1$ small enough we define the map

$$
T_{\lambda, \eta}: B_{r}^{\infty} \longrightarrow L_{t}^{2}\left(S^{2}\right) ; \quad \varepsilon \longmapsto T\left(\lambda_{\varepsilon}, \eta_{\varepsilon}, \Gamma_{\varepsilon}\right) .
$$

Then, for fixed $\lambda$ and $\eta$, the shape derivative of $T$ at $\Gamma$ is defined as the Fréchet derivative of $T_{\lambda, \eta}$ at 0 that we denote by $T_{\lambda, \eta}^{\prime}$. Moreover, we have the following characterization of $T_{\lambda, \eta}^{\prime}$.

Theorem 4.1. For an analytic boundary $\Gamma$ and two analytic functions $\lambda$ and $\eta$, the map $T_{\lambda, \eta}$ is Fréchet differentiable at 0 and its Fréchet derivative is given by

$$
T_{\lambda, \eta}^{\prime}(0) \cdot \varepsilon=U_{\varepsilon}^{\infty}
$$

where $U_{\varepsilon}^{\infty}$ is the far field pattern of the electric field that solves problem (1) with $f$ given by

$$
\begin{aligned}
f:= & -i k(\nu \cdot \varepsilon) \mathrm{H}_{T}+\operatorname{curl}_{\Gamma}[(\nu \cdot \varepsilon)(\nu \cdot \mathrm{E})]+\lambda(\nu \cdot \varepsilon)\left(2 R-2 H_{\Gamma} I d\right) \mathrm{H}_{T} \\
& -\lambda \nabla_{\Gamma}[(\nu \cdot \varepsilon)(\nu \cdot \mathrm{H})]+2 \operatorname{curl}_{\Gamma}\left[H_{\Gamma}(\nu \cdot \varepsilon) \eta \operatorname{curl}_{\Gamma}\left(\mathrm{H}_{T}\right)\right]+i k \mathcal{Z}\left[(\nu \cdot \varepsilon) \mathcal{Z} \mathrm{H}_{T}\right] \\
& +\left(\nabla_{\Gamma} \lambda \cdot \varepsilon\right) \mathrm{H}_{T}+\operatorname{curl}_{\Gamma}\left[\left(\nabla_{\Gamma} \eta \cdot \varepsilon\right) \operatorname{curl}_{\Gamma} \mathrm{H}_{T}\right],
\end{aligned}
$$

where $\mathcal{Z} \cdot=\operatorname{curl}_{\Gamma}\left(\eta \operatorname{curl}_{\Gamma} \cdot\right)+\lambda \cdot, R=\nabla_{\Gamma} \nu, 2 H_{\Gamma}=\operatorname{div}_{\Gamma} \nu, \mathrm{E}:=\mathscr{E}(\cdot, \hat{\theta}) \boldsymbol{p}$ and $\mathrm{H}:=\mathscr{H}(\cdot, \hat{\theta}) \boldsymbol{p}$.

Let us mention that this result coincide with the result obtained in [18, Theorem 3.4] for $\eta=0$ and $\lambda$ being constant $\left(\left(\nabla_{\Gamma} \lambda \cdot \varepsilon\right) \mathrm{H}_{T}=0\right)$.

Remark 4.2. We would like to emphasise that due to our definition of the shape derivative, its expression contains two non standard terms, namely $\left(\nabla_{\Gamma} \lambda \cdot \varepsilon\right) \mathrm{H}_{T}$ and $\operatorname{curl}_{\Gamma}\left[\left(\nabla_{\Gamma} \eta \cdot \varepsilon\right) \operatorname{curl}_{\Gamma} \mathrm{H}_{T}\right]$ that involve the tangential component of the perturbation $\varepsilon$. It has been numerically demonstrated in [6], for the scalar case, that these additional terms improve the speed of convergence of iterative schemes for simultaneous reconstruction of the impedance coefficients and obstacle shape.

Another possible choice for defining the shape derivative would be to first extend the impedance parameters $\lambda$ and $\eta$ in a neighbourhood of $\Gamma$ and then study the variation of $T(\lambda, \eta, \Gamma)$. In this case the expression of the shape derivative coincides with the one in Theorem 4.1 but without the above mentioned additional terms.

For the sake of completeness we also give the derivative of $T$ with respect to $\lambda$ and $\eta$, with $\Gamma$ being fixed. We introduce the functional

$$
T_{\Gamma}: \Lambda \longrightarrow L_{t}^{2}\left(S^{2}\right) ; \quad(\lambda, \eta) \longmapsto T(\lambda, \eta, \Gamma)
$$

where $\Lambda \subset\left(L^{\infty}(\Gamma)\right)^{2}$ is the subset of $(\lambda, \eta)$ satisfying the hypothesis of Theorem 2.1. The Fréchet derivative of $T_{\Gamma}$ is given below.

Theorem 4.3. The function $T_{\Gamma}$ is Fréchet differentiable on $\Lambda$ and for all $(\lambda, \eta) \in \Lambda$ its Fréchet derivative is the map $T_{\Gamma}^{\prime}(\lambda, \eta): L^{\infty}(\Gamma) \times L^{\infty}(\Gamma) \rightarrow\left(L_{t}^{2}\left(S^{2}\right)\right)^{3}$ defined by

$$
T_{\Gamma}^{\prime}(\lambda, \eta) \cdot(h, l)=U_{h, l}^{\infty} \quad \text { for all }(h, l) \in\left(L^{\infty}(\Gamma)\right)^{2},
$$

where $U_{h, l}^{\infty}$ is the far field pattern of the solution to problem (1) with

$$
f=-\left(\operatorname{curl}_{\Gamma}\left(l \operatorname{curl}_{\Gamma} \mathrm{H}_{T}\right)+h \mathrm{H}_{T}\right)
$$

and $\mathrm{H}:=\mathscr{H}^{s}(\cdot, \hat{\theta}) \boldsymbol{p}+\mathscr{H}^{i}(\cdot, \hat{\theta}) \boldsymbol{p}$.

Proof. The proof of this result is very similar to the proof of Proposition 6 in [7] in the scalar case and is not detailed here. See also [12]. 


\subsection{Proof of Theorem 4.1}

In this section we assume that $\lambda, \eta$ and $\Gamma$ are analytic and characterise the shape derivative of the far field pattern. We chose to follow the constructive procedure first proposed in [25] and then extended in [18] to treat the case of a classical impedance boundary condition ( $\eta=0$ and $\lambda$ being constant).

We recall that $\mathscr{E}^{s}(\cdot, \hat{\theta}) \mathbf{p}$ is the solution to problem (1) with $f$ being given by (2) for a given incident field $\left(\mathscr{E}^{i}(\cdot, \hat{\theta}) \mathbf{p}, \mathscr{H}^{i}(\cdot, \hat{\theta}) \mathbf{p}\right)$. Moreover, we denote $\left(\mathscr{E}_{\varepsilon}^{s}(\cdot, \hat{\theta}) \mathbf{p}, \mathscr{H}_{\varepsilon}^{s}(\cdot, \hat{\theta}) \mathbf{p}\right)$ the scattered field induced by the scattering of plane waves on $\Gamma_{\varepsilon}$ that solves

$$
\left\{\begin{array}{l}
\operatorname{curl} \mathrm{H}_{\varepsilon}+i k \mathrm{E}_{\varepsilon}=0 \quad \text { in } \Omega_{\mathrm{ext}}^{\varepsilon}, \\
\operatorname{curl} \mathrm{E}_{\varepsilon}-i k \mathrm{H}_{\varepsilon}=0 \quad \text { in } \Omega_{\mathrm{ext}}^{\varepsilon}, \\
\nu_{\varepsilon} \times \mathrm{E}_{\varepsilon}+\operatorname{curl}_{\Gamma_{\varepsilon}}\left(\eta_{\varepsilon} \operatorname{curl}_{\Gamma_{\varepsilon}} \mathrm{H}_{\varepsilon, T_{\varepsilon}}\right)+\lambda_{\varepsilon} \mathrm{H}_{\varepsilon, T_{\varepsilon}}=f \quad \text { on } \Gamma_{\varepsilon}, \\
\lim _{R \rightarrow \infty} \int_{\partial B_{R}}\left|\mathrm{H}_{\varepsilon} \times \hat{x}-\left(\hat{x} \times \mathrm{E}_{\varepsilon}\right) \times \hat{x}\right|^{2} d s=0,
\end{array}\right.
$$

where $\nu_{\varepsilon}$ is the outward normal to $\Gamma_{\varepsilon}, \Omega_{\text {ext }}^{\varepsilon}:=\mathbb{R}^{3} \backslash \bar{\Omega}_{\varepsilon}$ is the exterior domain to $\Omega_{\varepsilon}$ and $f$ is given by

$$
f:=-\left(\nu_{\varepsilon} \times \mathscr{E}^{i}(\cdot, \hat{\theta}) \mathbf{p}+\operatorname{curl}_{\Gamma}\left(\eta_{\varepsilon} \operatorname{curl}_{\Gamma} \mathscr{H}_{T_{\varepsilon}}^{i}(\cdot, \hat{\theta}) \mathbf{p}\right)+\lambda_{\varepsilon} \mathscr{H}_{T_{\varepsilon}}^{i}(\cdot, \hat{\theta}) \mathbf{p}\right)
$$

For all column vectors or matrices $V$ defined on $\Gamma_{\varepsilon}$, we denote $V_{T_{\varepsilon}}:=\left(\nu_{\varepsilon} \times V\right) \times \nu_{\varepsilon}$.

The scheme of the proof mainly consists in writing an appropriate integral representation formula for the difference $\mathscr{E}_{\varepsilon} s(\cdot, \hat{\theta}) \mathbf{p}-\mathscr{E}^{s}(\cdot, \hat{\theta}) \mathbf{p}$ on $\Gamma_{\varepsilon}$ and then performing an asymptotic expansion for small $\varepsilon$ of this integral representation. In the remaining of the section we do not mention the dependence of the electromagnetic fields on the position $x$, the direction of incidence $\hat{\theta}$ and the polarisation p. The notations $\left(\mathrm{E}^{s}, \mathrm{H}^{s}\right)$ and $\left(\mathrm{E}_{\varepsilon}^{s}, \mathrm{H}_{\varepsilon}^{s}\right)$ always refer to the scattered field associated with a plane wave of direction of incidence $\hat{\theta}$ and polarisation $\mathbf{p}$ given by (6). The notations $\mathcal{E}$ and $\mathcal{H}$ refer to the matrix representations of the total fields associated with the scattering of an electromagnetic dipole. To begin with, we assume that $\bar{\Omega} \subset \Omega_{\varepsilon}$ and give a first representation formula for $\mathrm{E}_{\varepsilon}^{s}-\mathrm{E}^{s}$.

Lemma 4.4. For any $z \in \mathbb{R}^{3} \backslash \overline{\Omega_{\varepsilon}}$,

$$
\mathrm{E}_{\varepsilon}^{s}(z)-\mathrm{E}^{s}(z)=-\int_{\Gamma_{\varepsilon}}\left[\nu_{\varepsilon} \times \mathcal{E}(y, z)+\operatorname{curl}_{\Gamma_{\varepsilon}}\left(\eta_{\varepsilon} \operatorname{curl}_{\Gamma_{\varepsilon}} \mathcal{H}_{T_{\varepsilon}}(y, z)\right)+\lambda_{\varepsilon} \mathcal{H}_{T_{\varepsilon}}(y, z)\right]^{\mathrm{T}} \mathrm{H}_{\varepsilon}(y) d s(y) .
$$

Proof. Let $z \in \mathbb{R}^{3} \backslash \overline{\Omega_{\varepsilon}}$. From the Stratton Chu integral representation (7) we first have

$$
\mathrm{E}^{s}(z)=\int_{\Gamma}\left\{\left[\mathcal{E}^{i}(y, z)\right]^{\mathrm{T}}\left[\nu(y) \times \mathrm{H}^{s}(y)\right]+\left[\mathcal{H}^{i}(y, z)\right]^{\mathrm{T}}\left[\nu(y) \times \mathrm{E}^{s}(y)\right]\right\} d s(y),
$$

which can be equivalently written as (using properties (9) and (10))

$$
\begin{aligned}
\mathrm{E}^{s}(z)= & -\int_{\Gamma}\left\{\left[\mathcal{E}^{s}(y, z)\right]^{\mathrm{T}}\left[\nu(y) \times \mathrm{H}^{i}(y)\right]+\left[\mathcal{H}^{s}(y, z)\right]^{\mathrm{T}}\left[\nu(y) \times \mathrm{E}^{i}(y)\right]\right\} d s(y) \\
& +\int_{\Gamma}\left\{[\mathcal{E}(y, z)]^{\mathrm{T}}[\nu(y) \times \mathrm{H}(y)]+[\mathcal{H}(y, z)]^{\mathrm{T}}[\nu(y) \times \mathrm{E}(y)]\right\} d s(y) .
\end{aligned}
$$

In this expression the second line is indeed equal to zero from the boundary conditions satisfied by $(\mathcal{E}, \mathcal{H})$ and $(\mathrm{E}, \mathrm{H})$ on $\Gamma$. Gauss' divergence theorem applied in $\Omega_{\varepsilon} \backslash \bar{\Omega}$ then implies

$$
\mathrm{E}^{s}(z)=-\int_{\Gamma_{\varepsilon}}\left\{\left[\mathcal{E}^{s}(y, z)\right]^{\mathrm{T}}\left[\nu_{\varepsilon}(y) \times \mathrm{H}^{i}(y)\right]+\left[\mathcal{H}^{s}(y, z)\right]^{\mathrm{T}}\left[\nu_{\varepsilon}(y) \times \mathrm{E}^{i}(y)\right]\right\} d s(y) .
$$

Using (10) we finally obtain

$$
\mathrm{E}^{s}(z)=-\int_{\Gamma_{\varepsilon}}\left\{\left[\mathcal{E}^{s}(y, z)\right]^{\mathrm{T}}\left[\nu_{\varepsilon}(y) \times \mathrm{H}_{\varepsilon}(y)\right]+\left[\mathcal{H}^{s}(y, z)\right]^{\mathrm{T}}\left[\nu_{\varepsilon}(y) \times \mathrm{E}_{\varepsilon}(y)\right]\right\} d s(y) .
$$

On the other hand, by Stratton-Chu formula (7) and by using (10) we have for $\mathrm{E}_{\varepsilon}^{s}$

$$
\mathrm{E}_{\varepsilon}^{s}(z)=\int_{\Gamma_{\varepsilon}}\left\{\left[\mathcal{E}^{i}(y, z)\right]^{\mathrm{T}}\left[\nu_{\varepsilon}(y) \times \mathrm{H}_{\varepsilon}(y)\right]+\left[\mathcal{H}^{i}(y, z)\right]^{\mathrm{T}}\left[\nu_{\varepsilon}(y) \times \mathrm{E}_{\varepsilon}(y)\right]\right\} d s(y) .
$$


By adding up the two last equations we obtain

$$
\mathrm{E}_{\varepsilon}^{s}(z)-\mathrm{E}^{s}(z)=\int_{\Gamma_{\varepsilon}}\left\{[\mathcal{E}(y, z)]^{\mathrm{T}}\left[\nu_{\varepsilon}(y) \times \mathrm{H}_{\varepsilon}(y)\right]+[\mathcal{H}(y, z)]^{\mathrm{T}}\left[\nu_{\varepsilon}(y) \times \mathrm{E}_{\varepsilon}(y)\right]\right\} d s(y) .
$$

We conclude the proof by using the boundary conditions satisfied by $\left(\mathrm{E}_{\varepsilon}, \mathrm{H}_{\varepsilon}\right)$ on $\Gamma_{\varepsilon}$ together with two integrations by part on $\Gamma_{\varepsilon}$.

We can actually substitute $\mathrm{H}_{\varepsilon}$ with $\mathrm{H}$ in formula (13) with an error of order $\|\varepsilon\|^{2}$. This relies of on the following continuity result.

Lemma 4.5. Let $B_{R}$ be a ball of radius $R$ sufficiently large so that $\bar{\Omega} \subset B_{R / 2}$. It exists $C_{R}>0$ such that for $\varepsilon \in B_{1}^{\infty}$ small enough we have

$$
\left\|\mathrm{E}_{\varepsilon} \circ f_{\varepsilon}-\mathrm{E}\right\|_{\left(H_{\text {curl }}\left(B_{R} \backslash \bar{\Omega}\right)\right)^{3}} \leq C_{R}\|\varepsilon\|
$$

and

$$
\left\|\mathrm{H}_{\varepsilon} \circ f_{\varepsilon}-\mathrm{H}\right\|_{\left(H_{\operatorname{curl}_{\Gamma}}(\Gamma)\right)^{3}}+\left\|\mathrm{H}_{\varepsilon} \circ f_{\varepsilon}-\mathrm{H}\right\|_{\left(H_{\text {curl }}\left(B_{R} \backslash \bar{\Omega}\right)\right)^{3}} \leq C_{R}\|\varepsilon\| .
$$

Proof. The proof of this result is rather straightforward. We only give here the two main ingredients. We first use that the forward problem has a unique solution that depends continuously on the boundary right hand side. We second use that the boundary operator $\operatorname{curl}_{\Gamma_{\varepsilon}} \eta_{\varepsilon} \operatorname{curl}_{\Gamma_{\varepsilon}}+\lambda_{\varepsilon}$ has a Lipschitz continuous dependence with respect to the perturbation $\varepsilon$. A detailed proof of a similar result in the case of Helmholtz' equation can be found in [5]. See also [12].

In the sequel we denote by $\mathcal{O}(\cdot)$ a $C^{\infty}([0,+\infty[)$ function for which there exists $C>0$ such that for all $x \in[0,+\infty[$

$$
|\mathcal{O}(x)| \leq C|x|
$$

Then by using the two previous lemmas, we obtain the following result.

Lemma 4.6. The following representation formula holds,

$$
\mathrm{E}_{\varepsilon}^{s}(z)-\mathrm{E}^{s}(z)=-\int_{\Gamma_{\varepsilon}}\left[\nu_{\varepsilon} \times \mathcal{E}(y, z)+\operatorname{curl}_{\Gamma_{\varepsilon}}\left(\eta_{\varepsilon} \operatorname{curl}_{\Gamma_{\varepsilon}} \mathcal{H}_{T_{\varepsilon}}(y, z)\right)+\lambda_{\varepsilon} \mathcal{H}_{T_{\varepsilon}}(y, z)\right]^{\mathrm{T}} \mathrm{H}(y) d s(y)+O\left(\|\varepsilon\|^{2}\right),
$$

uniformly for $z$ in a compact set $K \subset \mathbb{R}^{3} \backslash \overline{\Omega_{\varepsilon}}$.

Proof. The proof relies on the continuity result of Lemma 4.5. We refer to Lemma 4.3 in [6] in the scalar case and to [12].

Before we proceed any further with the proof of Theorem 4.1 we need to define an appropriate extension of $\lambda_{\varepsilon}, \eta_{\varepsilon}, \nu_{\varepsilon}$ and $\operatorname{curl}_{\Gamma_{\varepsilon}}$ in the domain $\Omega_{\varepsilon} \backslash \bar{\Omega}$. For any $x_{0} \in \Gamma$, there exists a local parametrisation of $\Gamma$, i.e. two open sets $U \subset \mathbb{R}^{2}$ and $V \subset \mathbb{R}^{3}$ which are neighbourhoods of 0 and $x_{0}$ respectively as well as a function $\varphi \in C^{1}(U ; V)$ such that $\varphi(0)=x_{0}$ and

$$
\Gamma \cap V=\{\varphi(\xi): \xi \in U\} .
$$

For $t \in[0,1]$ let us define

$$
f_{t}:=\mathrm{Id}+t \varepsilon, \quad \varphi_{t}:=f_{t} \circ \varphi
$$

For readability, the dependence of $f_{t}$ and $\varphi_{t}$ on $\varepsilon$ is not written explicitly. The function $\varphi_{t}$ defines a parametrisation of $\Gamma_{t}:=(\operatorname{Id}+t \varepsilon)(\Gamma)$ around $x_{0}^{t}:=f_{t}\left(x_{0}\right)$, and the vectors

$$
e_{j}^{t}:=\frac{\partial \varphi_{t}}{\partial \xi_{j}}=(\operatorname{Id}+t \nabla \varepsilon) \frac{\partial \varphi}{\partial \xi_{j}}=(\operatorname{Id}+t \nabla \varepsilon) e_{j}, \quad \text { for } j=1,2
$$

define a basis of tangent plane to $\Gamma_{t}$ at $x_{0}^{t}$ where $(\nabla \varepsilon)_{i, j}=\partial \varepsilon_{i} / \partial x_{j}$ is the Jacobian matrix of $\varepsilon$. We then define the associated covariant basis $\left(e_{t}^{i}\right)$ on $\Gamma_{t}$ at point $x_{0}^{t}$ by

$$
e_{t}^{i} \cdot e_{j}^{t}=\delta_{j}^{i}, \quad \text { pour } i, j=1,2 .
$$

The outward unit normal to $\Gamma_{t}$ at point $x_{0}^{t}$ is then given by

$$
\nu_{t}=\frac{e_{1}^{t} \times e_{2}^{t}}{\left|e_{1}^{t} \times e_{2}^{t}\right|}
$$


We extend the surface scalar curl by using the following formula

$$
\operatorname{curl}_{\Gamma_{t}}:=\nu_{t} \cdot \operatorname{curl}
$$

and for a row vector field or a matrix $V$ defined on $\Gamma_{t}$ we define

$$
V_{T_{t}}:=\left(\nu_{t} \times V\right) \times \nu_{t}
$$

the tangent component of $V$ on $\Gamma_{t}$. Finally, the impedance functions $\lambda_{\varepsilon}$ and $\eta_{\varepsilon}$ are extended in $\Omega_{\varepsilon} \backslash \bar{\Omega}$ in the following consistent way:

$$
\lambda_{t}:=\lambda \circ f_{t}^{-1}, \quad \eta_{t}:=\eta \circ f_{t}^{-1} .
$$

Let us recall the technical Lemma 4.5 from [6].

Lemma 4.7. Let $\lambda$ be $a C^{1}(\Gamma)$ function and define $\lambda_{t}:=\lambda \circ f_{t}^{-1}$. Then the following identity holds on $\Gamma$,

$$
\left.(\varepsilon \cdot \nu)\left(\nabla \lambda_{t} \cdot \nu_{t}\right)\right|_{t=0}=-\left(\nabla_{\Gamma} \lambda \cdot \varepsilon\right)
$$

Lemma 4.8. The representation formula of Lemma 4.6 yields

$$
\begin{gathered}
\mathrm{E}_{\varepsilon}^{s}(z)-\mathrm{E}^{s}(z)=-\int_{\Gamma}(\varepsilon \cdot \nu) \operatorname{div}\left\{[\mathcal{E} \times \mathrm{H}]^{\mathrm{T}}+\eta_{t}\left[\nu_{t} \operatorname{curl}_{\Gamma_{t}} \mathcal{H}_{T_{t}}\right]^{\mathrm{T}} \operatorname{curl}_{\Gamma_{t}} \mathrm{H}_{T_{t}}+\right. \\
\left.\lambda_{t}\left[\mathcal{H} \times\left(\nu_{t} \times \mathrm{H}\right)\right]^{\mathrm{T}}\right\}\left.\right|_{t=0} d s+\mathcal{O}\left(\|\varepsilon\|^{2}\right),
\end{gathered}
$$

uniformly for $z$ in a compact set $K \subset \mathbb{R}^{3} \backslash \overline{\Omega_{\varepsilon}}$.

Proof. From Lemma 4.6 we have

$$
\begin{gathered}
\mathrm{E}_{\varepsilon}^{s}(z)-\mathrm{E}^{s}(z)=-\int_{\Gamma_{\varepsilon}}\left[\nu_{\varepsilon} \times \mathcal{E}+\operatorname{curl}_{\Gamma_{\varepsilon}}\left(\eta_{\varepsilon} \operatorname{curl}_{\Gamma_{\varepsilon}} \mathcal{H}_{T_{\varepsilon}}\right)+\lambda_{\varepsilon} \mathcal{H}_{T_{\varepsilon}}\right]^{\mathrm{T}} \mathrm{H}(y) d s(y) \\
=-\int_{\Gamma_{\varepsilon}}\left\{[\mathcal{E} \times \mathrm{H}]^{\mathrm{T}} \nu_{\varepsilon}+\eta_{\varepsilon}\left[\operatorname{curl}_{\Gamma_{\varepsilon}} \mathcal{H}_{T_{\varepsilon}}\right]^{\mathrm{T}} \operatorname{curl}_{\Gamma_{\varepsilon}} \mathrm{H}_{T_{\varepsilon}}\right. \\
\left.\quad+\lambda_{\varepsilon}\left[\mathcal{H} \times\left(\nu_{\varepsilon} \times \mathrm{H}\right)\right]^{\mathrm{T}} \nu_{\varepsilon}\right\} d s+\mathcal{O}\left(\|\varepsilon\|^{2}\right) .
\end{gathered}
$$

The Gauss divergence theorem together with the boundary conditions satisfied by $(\mathcal{E}, \mathcal{H})$ on $\Gamma$ imply

$$
\begin{gathered}
\mathrm{E}_{\varepsilon}^{s}(z)-\mathrm{E}^{s}(z)=-\int_{\Omega_{\varepsilon} \backslash \bar{\Omega}} \operatorname{div}\left\{[\mathcal{E} \times \mathrm{H}]^{\mathrm{T}}+\eta_{t}\left[\nu_{t} \operatorname{curl}_{\Gamma_{t}} \mathcal{H}_{T_{t}}\right]^{\mathrm{T}} \operatorname{curl}_{\Gamma_{t}} \mathrm{H}_{T_{t}}+\right. \\
\left.\lambda_{t}\left[\mathcal{H} \times\left(\nu_{t} \times \mathrm{H}\right)\right]^{\mathrm{T}}\right\} d x+\mathcal{O}\left(\|\varepsilon\|^{2}\right) .
\end{gathered}
$$

By using the change of variable $\left(x_{\Gamma}, t\right) \mapsto x_{\Gamma}+t \varepsilon\left(x_{\Gamma}\right)$ between $\Gamma \times(0,1)$ and $\Omega_{\varepsilon} \backslash \bar{\Omega}$, and a Taylor expansion, this integral becomes after regrouping the $\mathcal{O}\left(\|\varepsilon\|^{2}\right)$ terms

$$
\begin{gathered}
\mathrm{E}_{\varepsilon}^{s}(z)-\mathrm{E}^{s}(z)=-\int_{\Gamma}(\varepsilon \cdot \nu) \operatorname{div}\left\{[\mathcal{E} \times \mathrm{H}]^{\mathrm{T}}+\eta_{t}\left[\nu_{t} \operatorname{curl}_{\Gamma_{t}} \mathcal{H}_{T_{t}}\right]^{\mathrm{T}} \operatorname{curl}_{\Gamma_{t}} \mathrm{H}_{T_{t}}+\right. \\
\left.\lambda_{t}\left[\mathcal{H} \times\left(\nu_{t} \times \mathrm{H}\right)\right]^{\mathrm{T}}\right\}\left.\right|_{t=0} d s+\mathcal{O}\left(\|\varepsilon\|^{2}\right) .
\end{gathered}
$$

In order to express the divergence term in Lemma 4.8 we need the following technical lemma which is proven in the appendix.

Lemma 4.9. For $V \in\left(C^{2}\left(\overline{\Omega_{\mathrm{ext}}}\right)\right)^{3}$,

$$
\left.(\varepsilon \cdot \nu) \operatorname{curl}\left(\nu_{t} \times V\right)\right|_{t=0}=(\varepsilon \cdot \nu)\left(\operatorname{div}_{\Gamma} V_{T}\right) \nu+(\varepsilon \cdot \nu)\left(R-2 H_{\Gamma} I d-\frac{\partial}{\partial \nu}\right) V_{T}+\left[\nabla_{\Gamma}(\nu \cdot \varepsilon) \times V\right] \times \nu
$$

and

$$
\left.(\varepsilon \cdot \nu) \frac{\partial}{\partial \nu}\left(\operatorname{curl}_{\Gamma_{t}} V_{T_{t}}\right)\right|_{t=0}=-(\varepsilon \cdot \nu) \operatorname{div}_{\Gamma}(\operatorname{curl} V)_{T}-2 H_{\Gamma}(\varepsilon \cdot \nu) \operatorname{curl}_{\Gamma} V_{T}-\nabla_{\Gamma}(\nu \cdot \varepsilon) \cdot(\operatorname{curl} V),
$$

where $R=\nabla_{\Gamma} \nu$ and $2 H_{\Gamma}=\operatorname{div}_{\Gamma} \nu$. 
We shall now use this lemma to express d the integral representation of $\mathrm{E}_{\varepsilon}^{s}-\mathrm{E}^{s}$ obtained in Lemma 4.8 in terms of boundary values of $\mathrm{E}$ and $\mathrm{H}$. We split the process into two parts: Lemma 4.10 and Lemma 4.11 .

Lemma 4.10. The following first identity holds for $(\mathcal{E}, \mathcal{H})$ and $(\mathrm{E}, \mathrm{H})$

$$
\begin{aligned}
\int_{\Gamma}(\nu \cdot \varepsilon) \operatorname{div}\{ & {\left.\left[\mathcal{E} \times \mathrm{H}+\lambda_{t} \mathcal{H} \times\left(\nu_{t} \times \mathrm{H}\right)\right]^{\mathrm{T}}\right\}\left.\right|_{t=0} d s=\int_{\Gamma} \mathcal{H}^{\mathrm{T}}\left\{i k(\nu \cdot \varepsilon) \mathrm{H}_{T}\right.} \\
& -i k \mathcal{Z}[(\nu \cdot \varepsilon)(\nu \times \mathrm{E})]-\operatorname{curl}_{\Gamma}[(\nu \cdot \varepsilon)(\nu \cdot \mathrm{E})]-\lambda(\nu \cdot \varepsilon)\left(2 R-2 H_{\Gamma}\right) \mathrm{H}_{T} \\
& \left.-i k \lambda(\nu \cdot \varepsilon) \mathcal{Z} \mathrm{H}_{T}+\lambda \nabla_{\Gamma}[(\nu \cdot \varepsilon)(\nu \cdot \mathrm{H})]-i k \mathcal{Z}\left[\lambda(\nu \cdot \varepsilon) \mathrm{H}_{T}\right]\right\} d s .
\end{aligned}
$$

Proof. Let us first recall that for two vector fields $V$ and $W$ and for a function $\varphi$,

$$
\operatorname{div}(V \times W)=\operatorname{curl} V \cdot W-V \cdot \operatorname{curl} W, \quad \operatorname{curl}(\varphi V)=\nabla \varphi \times V+\varphi \operatorname{curl} V .
$$

Therefore, from Maxwell's equations

$$
\left.\operatorname{div}\left([\mathcal{E} \times \mathrm{H}]^{\mathrm{T}}\right)\right|_{t=0}=\left.i k\left(\mathcal{H}^{\mathrm{T}} \mathrm{H}+\mathcal{E}^{\mathrm{T}} \mathrm{E}\right)\right|_{\Gamma} .
$$

From the boundary conditions satisfied by $(\mathrm{E}, \mathrm{H})$ and the formula $\operatorname{curl}_{\Gamma} V=\nu \cdot \operatorname{curl} V$ we have

$$
\mathcal{E}^{\mathrm{T}} \mathrm{E}=[\nu \times \mathcal{E}]^{\mathrm{T}}(\nu \times \mathrm{E})+(\nu \cdot \mathrm{E})\left[\mathcal{E}^{\mathrm{T}} \nu\right]=-\left[\mathcal{Z} \mathcal{H}_{T}\right]^{\mathrm{T}}(\nu \times \mathrm{E})-\frac{1}{i k}(\nu \cdot \mathrm{E})\left[\operatorname{curl}_{\Gamma} \mathcal{H}_{T}\right]^{\mathrm{T}}
$$

where $\mathcal{Z}=\operatorname{curl}_{\Gamma} \eta \operatorname{curl}_{\Gamma}+\lambda$. Similarly

$$
\begin{gathered}
\left.\operatorname{div}\left\{\lambda_{t}\left[\mathcal{H} \times\left(\nu_{t} \times \mathrm{H}\right)\right]^{T}\right\}\right|_{t=0}=\left.\left[\left.\operatorname{curl} \mathcal{H}\right|_{\Gamma}\right]^{\mathrm{T}}\left(\lambda_{t} \nu \times \mathrm{H}\right)\right|_{\Gamma}-\left.\left.\mathcal{H}^{\mathrm{T}}\right|_{\Gamma} \operatorname{curl}\left[\lambda_{t}\left(\nu_{t} \times \mathrm{H}\right)\right]\right|_{t=0} \\
=-\left.i k \lambda\left[\mathcal{E}^{\mathrm{T}}(\nu \times \mathrm{H})\right]\right|_{\Gamma}-\left.\left.\mathcal{H}^{\mathrm{T}}\right|_{\Gamma} \operatorname{curl}\left[\nu_{t} \times\left(\lambda_{t} \mathrm{H}\right)\right]\right|_{t=0} .
\end{gathered}
$$

By using the first formula of Lemma 4.9 we obtain

$$
\begin{aligned}
\left.\operatorname{div}\left\{\lambda_{t}\left[\mathcal{H} \times\left(\nu_{t} \times \mathrm{H}\right)\right]^{\mathrm{T}}\right\}\right|_{t=0}=-\left.i k \lambda \mathcal{E}^{\mathrm{T}}(\nu \times \mathrm{H})\right|_{\Gamma} \\
\quad-\mathcal{H}^{\mathrm{T}}\left\{\operatorname{div}_{\Gamma}\left(\lambda \mathrm{H}_{T}\right) \nu+\left.\left(R-2 H_{\Gamma}-\frac{\partial}{\partial \nu}\right)\left(\lambda_{t} \mathrm{H}_{T}\right)\right|_{t=0}+\frac{\lambda}{\nu \cdot \varepsilon}\left[\nabla_{\Gamma}(\nu \cdot \varepsilon) \times \mathrm{H}\right] \times \nu\right\}
\end{aligned}
$$

and by using the boundary conditions

$$
\begin{aligned}
\operatorname{div}\left\{\lambda_{t}\right. & {\left.\left[\mathcal{H} \times\left(\nu_{t} \times \mathrm{H}\right)\right]^{\mathrm{T}}\right\}\left.\right|_{t=0}=-\left.i k \lambda\left[\left(\mathcal{Z} \mathcal{H}_{T}\right)^{\mathrm{T}} \mathrm{H}_{T}\right]\right|_{\Gamma} } \\
& -\mathcal{H}^{\mathrm{T}}\left\{\operatorname{div}_{\Gamma}\left(\lambda \mathrm{H}_{T}\right) \nu+\left.\left(R-2 H_{\Gamma}-\frac{\partial}{\partial \nu}\right)\left(\lambda_{t} \mathrm{H}_{T}\right)\right|_{t=0}+\frac{\lambda}{\nu \cdot \varepsilon}\left[\nabla_{\Gamma}(\nu \cdot \varepsilon) \times \mathrm{H}\right] \times \nu\right\} .
\end{aligned}
$$

We expand $\left.\frac{\partial\left(\lambda_{t} \mathrm{H}_{T}\right)}{\partial \nu}\right|_{t=0}$ by using Lemma 4.7

$$
\begin{aligned}
\operatorname{div}\left\{\lambda_{t}\right. & {\left.\left.\left[\mathcal{H} \times\left(\nu_{t} \times \mathrm{H}\right)\right]^{\mathrm{T}}\right\}\left.\right|_{t=0}=-i k \lambda\left(\mathcal{Z} \mathcal{H}_{T}\right)^{\mathrm{T}} \mathrm{H}_{T}\right)-\frac{\nabla_{\Gamma} \lambda \cdot \varepsilon}{\nu \cdot \varepsilon} \mathcal{H}^{\mathrm{T}} \mathrm{H}_{T} } \\
& -\mathcal{H}^{\mathrm{T}}\left\{\operatorname{div}_{\Gamma}\left(\lambda \mathrm{H}_{T}\right) \nu+\lambda\left(R-2 H_{\Gamma}-\frac{\partial}{\partial \nu}\right) \mathrm{H}_{T}+\frac{\lambda}{\nu \cdot \varepsilon}\left[\nabla_{\Gamma}(\nu \cdot \varepsilon) \times \mathrm{H}\right] \times \nu\right\} .
\end{aligned}
$$

From [28, formula 2.5.225]

$$
-\frac{\partial}{\partial \nu} \mathrm{H}_{T}=\nu \times \operatorname{curl} \mathrm{H}-\nabla_{\Gamma}(\nu \cdot \mathrm{H})+R \mathrm{H}_{T},
$$

we get, using the boundary conditions,

$$
-\frac{\partial}{\partial \nu} \mathrm{H}_{T}=i k \mathcal{Z} \mathrm{H}_{T}-\nabla_{\Gamma}(\nu \cdot \mathrm{H})+R \mathrm{H}_{T} .
$$

The identity $U \times(V \times W)=(U \cdot W) V-(U \cdot V) W$ yields

$$
-\lambda\left[\nabla_{\Gamma}(\nu \cdot \varepsilon) \times \mathrm{H}\right] \times \nu=\lambda\left(\nabla_{\Gamma}(\nu \cdot \varepsilon)\right)(\nu \cdot \mathrm{H}),
$$

whence

$$
-\lambda\left[\nabla_{\Gamma}(\nu \cdot \varepsilon) \times \mathrm{H}\right] \times \nu+\lambda(\nu \cdot \varepsilon) \nabla_{\Gamma}(\nu \cdot \mathrm{H})=\lambda \nabla_{\Gamma}[(\nu \cdot \varepsilon)(\nu \cdot \mathrm{H})] .
$$


We finally get, using the symmetry of $\mathcal{Z}$,

$$
\begin{aligned}
\int_{\Gamma}(\nu \cdot \varepsilon) \operatorname{div}\{ & {\left.\left[\mathcal{E} \times \mathrm{H}+\lambda_{t} \mathcal{H} \times\left(\nu_{t} \times \mathrm{H}\right)\right]^{\mathrm{T}}\right\}\left.\right|_{t=0} d s=\int_{\Gamma} \mathcal{H}^{\mathrm{T}}\left\{i k(\nu \cdot \varepsilon) \mathrm{H}-(\nu \cdot \varepsilon) \operatorname{div}_{\Gamma}\left(\lambda \mathrm{H}_{T}\right) \nu\right.} \\
& -i k \mathcal{Z}[(\nu \cdot \varepsilon)(\nu \times \mathrm{E})]-\operatorname{curl}_{\Gamma}[(\nu \cdot \varepsilon)(\nu \cdot \mathrm{E})]-\lambda(\nu \cdot \varepsilon)\left(2 R-2 H_{\Gamma}\right) \mathrm{H}_{T} \\
& \left.-i k \lambda(\nu \cdot \varepsilon) \mathcal{Z} \mathrm{H}_{T}+\lambda \nabla_{\Gamma}[(\nu \cdot \varepsilon)(\nu \cdot \mathrm{H})]-i k \mathcal{Z}\left[\lambda(\nu \cdot \varepsilon) \mathrm{H}_{T}\right]-\left(\nabla_{\Gamma} \lambda \cdot \varepsilon\right) \mathrm{H}_{T}\right\} d s .
\end{aligned}
$$

We conclude the proof by noticing that, using Maxwell's equations,

$$
i k(\nu \cdot \mathrm{H})-\operatorname{div}_{\Gamma}\left(\lambda \mathrm{H}_{T}\right)=\left(-\operatorname{div}_{\Gamma}(\nu \times \mathrm{E})-\operatorname{div}_{\Gamma}\left(\lambda \mathrm{H}_{T}\right)\right)=\operatorname{div}_{\Gamma}\left(\operatorname{curl}_{\Gamma}\left(\eta \operatorname{curl}_{\Gamma} \mathrm{H}_{T}\right)\right)=0
$$

since $\operatorname{div}_{\Gamma} \operatorname{curl}_{\Gamma}=0$.

Lemma 4.11. The following second identity holds for $(\mathcal{E}, \mathcal{H})$ and $(\mathrm{E}, \mathrm{H})$

$$
\begin{aligned}
\int_{\Gamma}(\nu \cdot \varepsilon) & \left.\operatorname{div}\left\{\eta_{t}\left[\nu_{t} \operatorname{curl}_{\Gamma_{t}} \mathcal{H}_{T_{t}}\right]^{\mathrm{T}} \operatorname{curl}_{\Gamma_{t}} \mathrm{H}_{T_{t}}\right\}\right|_{t=0} d s=\int_{\Gamma} \mathcal{H}^{\mathrm{T}}\left\{-2 \operatorname{curl}_{\Gamma}\left[H_{\Gamma}(\nu \cdot \varepsilon) \eta \operatorname{curl} l_{\Gamma}\left(\mathrm{H}_{T}\right)\right]\right. \\
& -i k \mathcal{Z}\left[(\nu \cdot \varepsilon) \operatorname{curl}_{\Gamma}\left(\eta \operatorname{curl}_{\Gamma} \mathrm{H}_{T}\right)\right]-i k \operatorname{curl}_{\Gamma}\left[\eta \operatorname{curl}_{\Gamma}\left[(\nu \cdot \varepsilon) \mathcal{Z} \mathrm{H}_{T}\right]\right] \\
& \left.\left.-\operatorname{curl}_{\Gamma}\left[\left(\nabla_{\Gamma} \eta \cdot \varepsilon\right) \operatorname{curl}_{\Gamma} \mathrm{H}_{T}\right]\right\} d s\right\} d s .
\end{aligned}
$$

Proof. First of all

$$
\begin{aligned}
\operatorname{div}\left\{\eta_{t}\left[\nu_{t} \operatorname{curl}_{\Gamma_{t}} \mathcal{H}_{T_{t}}\right]^{\mathrm{T}}\right. & \left.\operatorname{curl}_{\Gamma_{t}} \mathrm{H}_{T_{t}}\right\}\left.\right|_{t=0}=2 H_{\Gamma} \eta \operatorname{curl}_{\Gamma} \mathrm{H}_{T}\left[\operatorname{curl}_{\Gamma} \mathcal{H}_{T}\right]^{\mathrm{T}} \\
& +\left.\frac{\partial \eta_{t}}{\partial \nu}\right|_{t=0} \operatorname{curl}_{\Gamma} \mathrm{H}_{T}\left[\operatorname{curl}_{\Gamma} \mathcal{H}_{T}\right]^{\mathrm{T}}+\left.\eta \frac{\partial}{\partial \nu}\left(\operatorname{curl}_{\Gamma_{t}} \mathrm{H}_{T_{t}}\left[\operatorname{curl}_{\Gamma_{t}} \mathcal{H}_{T_{t}}\right]^{\mathrm{T}}\right)\right|_{t=0}
\end{aligned}
$$

where by using Lemma 4.7 once more we have

$$
\left.\frac{\partial \eta_{t}}{\partial \nu}\right|_{t=0}=\frac{\nabla_{\Gamma} \eta \cdot \varepsilon}{\nu \cdot \varepsilon}
$$

From the second equality in Lemma 4.9 we have by using Maxwell's equations

$$
\begin{aligned}
\left.\frac{\partial}{\partial \nu}\left(\operatorname{curl}_{\Gamma_{t}} \mathrm{H}_{T_{t}} \operatorname{curl}_{\Gamma_{t}} \mathcal{H}_{T_{t}}\right)\right|_{t=0} & =\left.\operatorname{curl}_{\Gamma} \mathrm{H}_{T} \frac{\partial}{\partial \nu}\left(\operatorname{curl}_{\Gamma_{t}} \mathcal{H}_{T_{t}}\right)\right|_{t=0}+\left.\operatorname{curl}_{\Gamma} \mathcal{H}_{T} \frac{\partial}{\partial \nu}\left(\operatorname{curl}_{\Gamma_{t}} \mathrm{H}_{T_{t}}\right)\right|_{t=0} \\
= & \operatorname{curl}_{\Gamma} \mathrm{H}_{T}\left\{i k \operatorname{div}_{\Gamma} \mathcal{E}_{T}-2 H_{\Gamma} \operatorname{curl}_{\Gamma} \mathcal{H}_{T}+\frac{i k}{\nu \cdot \varepsilon} \mathcal{E}^{\mathrm{T}} \nabla_{\Gamma}(\nu \cdot \varepsilon)\right\} \\
& +\operatorname{curl}_{\Gamma} \mathcal{H}_{T}\left\{i k \operatorname{div}_{\Gamma} \mathrm{E}_{T}-2 H_{\Gamma} \operatorname{curl}_{\Gamma} \mathrm{H}_{T}+\frac{i k}{\nu \cdot \varepsilon} \nabla_{\Gamma}(\nu \cdot \varepsilon) \cdot \mathrm{E}\right\}
\end{aligned}
$$

The boundary conditions satisfied by $(\mathrm{E}, \mathrm{H})$ and $(\mathcal{E}, \mathcal{H})$ implies

$$
\begin{aligned}
\left.\frac{\partial}{\partial \nu}\left(\operatorname{curl}_{\Gamma_{t}} \mathrm{H}_{T_{t}} \operatorname{curl}_{\Gamma_{t}} \mathcal{H}_{T_{t}}\right)\right|_{t=0} & \\
= & \operatorname{curl}_{\Gamma} \mathrm{H}_{T}\left\{-i k \operatorname{curl}_{\Gamma}\left(\mathcal{Z} \mathcal{H}_{T}\right)-2 H_{\Gamma} \operatorname{curl}_{\Gamma} \mathcal{H}_{T}+\frac{i k}{\nu \cdot \varepsilon}\left[\mathcal{Z} \mathcal{H}_{T}\right]^{\mathrm{T}} \operatorname{curl}_{\Gamma}(\nu \cdot \varepsilon)\right\} \\
& +\operatorname{curl}_{\Gamma} \mathcal{H}_{T}\left\{-i k \operatorname{curl}_{\Gamma}\left(\mathcal{Z} \mathrm{H}_{T}\right)-2 H_{\Gamma} \operatorname{curl}_{\Gamma} \mathrm{H}_{T}+\frac{i k}{\nu \cdot \varepsilon}\left(\mathcal{Z} \mathrm{H}_{T}\right) \cdot \operatorname{curl}_{\Gamma}(\nu \cdot \varepsilon)\right\}
\end{aligned}
$$

We recall that for a function $\varphi$ and a vector field $V$ we have $\operatorname{curl}_{\Gamma}(\varphi V)=-V \cdot \operatorname{curl}_{\Gamma}(\varphi)+\varphi \operatorname{curl}_{\Gamma}(V)$, therefore the previous relation gives

$$
\begin{aligned}
\left.\frac{\partial}{\partial \nu}\left(\operatorname{curl}_{\Gamma_{t}} \mathrm{H}_{T_{t}} \operatorname{curl}_{\Gamma_{t}} \mathcal{H}_{T_{t}}\right)\right|_{t=0}=-4 H_{\Gamma} \operatorname{curl}_{\Gamma} \mathcal{H}_{T} \operatorname{curl}_{\Gamma} \mathrm{H}_{T} \\
-\frac{i k}{\nu \cdot \varepsilon} \operatorname{curl}_{\Gamma}\left[(\nu \cdot \varepsilon) \mathcal{Z} \mathcal{H}_{T}\right] \operatorname{curl}_{\Gamma} \mathrm{H}_{T}-\frac{i k}{\nu \cdot \varepsilon} \operatorname{curl}_{\Gamma} \mathcal{H}_{T} \operatorname{curl}_{\Gamma}\left[(\nu \cdot \varepsilon) \mathcal{Z} \mathrm{H}_{T}\right] .
\end{aligned}
$$

Therefore

$$
\begin{aligned}
\int_{\Gamma}(\nu \cdot \varepsilon) & \left.\operatorname{div}\left\{\eta_{t}\left[\nu_{t} \operatorname{curl}_{\Gamma_{t}} \mathcal{H}_{T_{t}}\right]^{\mathrm{T}} \operatorname{curl}_{\Gamma_{t}} \mathrm{H}_{T_{t}}\right\}\right|_{t=0} d s=\int_{\Gamma}\left\{-2 H_{\Gamma}(\nu \cdot \varepsilon) \eta\left[\operatorname{curl}_{\Gamma} \mathcal{H}_{T}\right]^{\mathrm{T}} \operatorname{curl}_{\Gamma} \mathrm{H}_{T}\right. \\
& -i k \eta\left[\operatorname{curl}_{\Gamma}\left[(\nu \cdot \varepsilon) \mathcal{Z} \mathcal{H}_{T}\right]\right]^{\mathrm{T}} \operatorname{curl}_{\Gamma} \mathrm{H}_{T}-i k \eta\left[\operatorname{curl}_{\Gamma} \mathcal{H}_{T}\right]^{\mathrm{T}} \operatorname{curl}_{\Gamma}\left[(\nu \cdot \varepsilon) \mathcal{Z} \mathrm{H}_{T}\right] \\
& \left.-\left(\nabla_{\Gamma} \eta \cdot \varepsilon\right)\left[\operatorname{curl}_{\Gamma} \mathcal{H}_{T}\right]^{\mathrm{T}} \operatorname{curl}_{\Gamma} \mathrm{H}_{T}\right\} d s
\end{aligned}
$$

and integration by part on $\Gamma$ gives the final expression. 
Gathering the results of Lemmas 4.10 and 4.11 we obtain the following proposition.

Proposition 4.12. We have the following formula for the discrepancy between $\mathrm{E}_{\varepsilon}^{s}$ and $\mathrm{E}^{s}$ :

$$
\mathrm{E}_{\varepsilon}^{s}(z)-\mathrm{E}^{s}(z)=\int_{\Gamma} \mathcal{H}(\cdot, z)^{\mathrm{T}} B_{\varepsilon}(\mathrm{E}, \mathrm{H}) d s+\mathcal{O}\left(\|\varepsilon\|^{2}\right)
$$

uniformly for $z$ in a compact set $K \subset \mathbb{R}^{3} \backslash \overline{\Omega_{\varepsilon}}$ where

$$
\begin{aligned}
B_{\varepsilon}(\mathrm{E}, \mathrm{H}):= & -i k(\nu \cdot \varepsilon) \mathrm{H}_{T}+\operatorname{curl}_{\Gamma}[(\nu \cdot \varepsilon)(\nu \cdot \mathrm{E})]+\lambda(\nu \cdot \varepsilon)\left(2 R-2 H_{\Gamma}\right) \mathrm{H}_{T} \\
& -\lambda \nabla_{\Gamma}[(\nu \cdot \varepsilon)(\nu \cdot \mathrm{H})]+2 \operatorname{curl}_{\Gamma}\left[H_{\Gamma}(\nu \cdot \varepsilon) \eta \operatorname{curl}_{\Gamma} \mathrm{H}_{T}\right]+i k \mathcal{Z}\left[(\nu \cdot \varepsilon) \mathcal{Z H}_{T}\right] \\
& +\left(\nabla_{\Gamma} \lambda \cdot \varepsilon\right) \mathrm{H}_{T}+\operatorname{curl}_{\Gamma}\left[\left(\nabla_{\Gamma} \eta \cdot \varepsilon\right) \operatorname{curl}_{\Gamma} \mathrm{H}_{T}\right] .
\end{aligned}
$$

We recall that $\mathcal{Z} \cdot=\operatorname{curl}_{\Gamma}\left(\eta \operatorname{curl}_{\Gamma} \cdot\right)+\lambda \cdot, R=\nabla_{\Gamma} \nu$ and $2 H_{\Gamma}=\operatorname{div}_{\Gamma} \nu$.

Proof. First of all, by Lemmas 4.10 and 4.11:

$$
\begin{aligned}
\int_{\Gamma} & \left.(\varepsilon \cdot \nu) \operatorname{div}\left\{\mathcal{E} \times \mathrm{H}+\lambda \mathcal{H} \times\left(\nu_{t} \times \mathrm{H}\right)+\eta \nu_{t}\left(\operatorname{curl}_{\Gamma_{t}} \mathrm{H}_{T_{t}} \operatorname{curl}_{\Gamma_{t}} \mathcal{H}_{T_{t}}\right)\right\}\right|_{t=0} d s \\
\quad & =\int_{\Gamma} \mathcal{H}^{\mathrm{T}}\left\{i k(\nu \cdot \varepsilon) \mathrm{H}_{T}-i k \mathcal{Z}[(\nu \cdot \varepsilon) \nu \times \mathrm{E})\right]-\operatorname{curl}_{\Gamma}[(\nu \cdot \varepsilon)(\nu \cdot \mathrm{E})]-\lambda(\nu \cdot \varepsilon)\left(2 R-2 H_{\Gamma}\right) \mathrm{H}_{T} \\
& -i k \lambda(\nu \cdot \varepsilon) \mathcal{Z} \mathrm{H}_{T}+\lambda \nabla_{\Gamma}[(\nu \cdot \varepsilon)(\nu \cdot \mathrm{H})]-i k \mathcal{Z}\left[\lambda(\nu \cdot \varepsilon) \mathrm{H}_{T}\right]-2 \operatorname{curl}_{\Gamma}\left[H_{\Gamma}(\nu \cdot \varepsilon) \eta \operatorname{curl}_{\Gamma} \mathrm{H}_{T}\right] \\
& \left.-i k \mathcal{Z}\left[(\nu \cdot \varepsilon) \operatorname{curl}_{\Gamma}\left(\eta \operatorname{curl}_{\Gamma} \mathrm{H}_{T}\right)\right]-i k \operatorname{curl}_{\Gamma}\left[\eta \operatorname{curl}_{\Gamma}\left[(\nu \cdot \varepsilon) \mathcal{Z} \mathrm{H}_{T}\right]\right]\right\} d s .
\end{aligned}
$$

The boundary conditions satisfied by $(\mathrm{E}, \mathrm{H})$ implies that

$$
-i k \mathcal{Z}[(\nu \cdot \varepsilon)(\nu \times \mathrm{E})]-i k \mathcal{Z}\left[\lambda(\nu \cdot \varepsilon) \mathrm{H}_{T}\right]-i k \mathcal{Z}\left[(\nu \cdot \varepsilon) \operatorname{curl}_{\Gamma}\left(\eta \operatorname{curl}_{\Gamma} \mathrm{H}_{T}\right)\right]=0 .
$$

We also have

$$
-i k \operatorname{curl}_{\Gamma}\left[\eta \operatorname{curl}_{\Gamma}\left[(\nu \cdot \varepsilon) \mathcal{Z}\left(\mathrm{H}_{T}\right)\right]\right]-i k \lambda(\nu \cdot \varepsilon) \mathcal{Z} \mathrm{H}_{T}=-i k \mathcal{Z}\left[(\nu \cdot \varepsilon) \mathcal{Z} \mathrm{H}_{T}\right] .
$$

We then obtain the result by using Lemma 4.8 .

We now have all the necessary results to conclude the proof of Theorem 4.1.

Proof of Theorem 4.1. First of all, since $\lambda, \eta$ and $\Gamma$ are analytical, we can extend Proposition 4.12 to the case where $\Omega$ is not included in $\Omega_{\varepsilon}$ (see [18] for the details). Moreover, by using the Stratton-Chu representation formula (7) we prove that for $f \in H_{\operatorname{curl}_{\Gamma}}(\Gamma)^{*}$, the electric field $\mathrm{E}_{f}$ solution to problem (1) is such that

$$
\mathrm{E}_{f}(z)=\int_{\Gamma} \mathcal{H}(y, z)^{\mathrm{T}} f(y) d s(y) \text { for all } z \in \Omega_{\mathrm{ext}} .
$$

Therefore, Proposition 4.12 together with formula (16) concludes the proof.

\subsection{Numerical validation of the shape derivative}

In this section we describe the numerical procedure we use to compute the shape derivative of the scattered field. We also present numerical experiments that we use to validate the expression of the shape derivative given in Theorem 4.1. To be more precise we compute the shape derivative of the following $L^{2}$ cost functional

$$
F(\Gamma):=\frac{1}{2}\left\|T(\lambda, \eta, \Gamma)-\mathrm{E}_{\mathrm{obs}}^{\infty}\right\|_{L_{t}^{2}\left(S^{2}\right)}^{2} \cdot
$$

where $\mathrm{E}_{\mathrm{obs}}^{\infty}:=T\left(\lambda, \eta, \Gamma_{\mathrm{obs}}\right)$ for given shape $\Gamma_{\mathrm{obs}}$, impedance coefficients $\lambda$ and $\eta$, direction of incidence $\hat{\theta}$ and polarisation $\mathbf{p}$. In the next section we show how to solve a shape identification problem by minimizing such cost functional.

For analytic $\lambda, \eta$ and $\Gamma$, by using the result of Theorem 4.1 and since the forward problem is linear we prove that the shape derivative of $F$ in the direction $\varepsilon$ is given by

$$
F^{\prime}(\Gamma) \cdot \varepsilon=\frac{1}{4 \pi} \Re\left(\int_{\Gamma} \mathcal{G}(y) \cdot B_{\varepsilon}(\mathrm{E}, \mathrm{H})(y) d s(y)\right),
$$


where $B_{\varepsilon}$ is given in Proposition 4.12, $\mathrm{E}(y)=\mathscr{E}^{s}(y, \hat{\theta}) \mathbf{p}+\mathscr{E}^{i}(y, \hat{\theta}) \mathbf{p}, \mathrm{H}(y)=\mathscr{H}^{s}(y, \hat{\theta}) \mathbf{p}+\mathscr{H}^{i}(y, \hat{\theta}) \mathbf{p}$ and the adjoint state $\mathcal{G}$ is given by

$$
\mathcal{G}(y):=\int_{S^{2}} \mathscr{H}(y,-\hat{x}) \mathbf{g}(\hat{x}) d s(\hat{x}),
$$

where

$$
\mathbf{g}(\hat{x}):=\left(\overline{T(\lambda, \eta, \Gamma)-\mathrm{E}_{\mathrm{obs}}^{\infty}}\right)(\hat{x}) .
$$

From (17), evaluating the shape derivative of $F$ requires the knowledge of two total fields: (E, H) and $\mathcal{G}$ that we numerically compute by solving two scattering problems: a first one associated with the incident plane wave $\left(\mathscr{E}^{i}(y, \hat{\theta}) \mathbf{p}, \mathscr{H}^{i}(y, \hat{\theta}) \mathbf{p}\right)$ and a second one associated with an electromagnetic Herglotz wave $\left(\mathcal{G}_{\mathrm{E}}^{i}, \mathcal{G}_{\mathrm{H}}^{i}\right)$ given by

$$
\mathcal{G}_{\mathrm{E}}^{i}(y):=\int_{S^{2}} \mathrm{E}^{i}(y,-\hat{x}) \mathbf{g}(\hat{x}) d s(\hat{x}), \quad \mathcal{G}_{\mathrm{H}}^{i}(y):=\int_{S^{2}} \mathrm{H}^{i}(y,-\hat{x}) \mathbf{g}(\hat{x}) d s(\hat{x}) .
$$

Then $\mathcal{G}=\mathcal{G}_{\mathrm{H}}^{i}+\mathcal{G}_{\mathrm{H}}^{s}$ is the total magnetic field associated with the solution $\left(\mathcal{G}_{\mathrm{E}}^{s}, \mathcal{G}_{\mathrm{H}}^{s}\right)$ to (1) for $f$ being given by $(2)$ with $\mathrm{E}^{i}=\mathcal{G}_{\mathrm{E}}^{i}$ and $\mathrm{H}^{i}=\mathcal{G}_{\mathrm{H}}^{i}$.

We choose here to use edge finite elements to solve the second order system associated with the Maxwell system (1) which can be written as

$$
\left\{\begin{array}{l}
\operatorname{curl} \operatorname{curl~} \mathrm{H}^{s}-k^{2} \mathrm{H}^{s}=0 \quad \text { in } \Omega_{\mathrm{ext}}, \\
\frac{i}{k} \nu \times \operatorname{curl~} \mathrm{H}^{s}+\operatorname{curl}_{\Gamma}\left(\eta \operatorname{curl}_{\Gamma} \mathrm{H}_{T}^{s}\right)+\lambda \mathrm{H}_{T}^{s}=f \quad \text { on, } \Gamma
\end{array}\right.
$$

where $f$ is given by (2) in term of the incident field $\left(\mathrm{E}^{i}, \mathrm{H}^{i}\right)$. We complement this system with the approximate first order radiation condition

$$
\hat{x} \times \operatorname{curl} \mathrm{H}^{s}+i k\left(\hat{x} \times \mathrm{H}^{s}\right) \times \hat{x}=0 \text { on } \Sigma,
$$

where $\Sigma$ is is closed regular surface that is far from $\Gamma$. Typically, $\Sigma$ is a sphere or an ellipsoid such that the distance between $\Gamma$ and $\Sigma$ is of the order of one wavelength. We discretise the domain contained between $\Sigma$ and $\Gamma$ with tetrahedron with approximately twelve tetrahedron per wavelength $(l:=2 \pi / k)$ and we approximate the field $\mathrm{H}$ with the Nédélec edge elements of the first kind (see [27]). We use the finite elements software Freefem++ (see [20]) to set up and solve the discrete system. Finally, the farfield pattern is computed by using the integral representation formula (8). The solver has been validated against Mie's serie solutions in the case of the scattering by a sphere and we obtained relative errors smaller than $5 \%$ on the $L^{2}$ norm of the far field pattern in several test cases. Then we evaluate (17) by using numerical integration. The only problematic point would be the numerical evaluation of the operator $B_{\varepsilon}$ since it contains fourth order derivatives. First we transform (17) by using integration by part on $\Gamma$ :

$$
\begin{aligned}
F^{\prime}(\Gamma) \cdot \varepsilon & =\frac{1}{4 \pi} \Re \int_{\Gamma}(\nu \cdot \varepsilon)\left\{-i k \mathrm{H}_{T} \cdot \mathcal{G}+(\nu \cdot \mathrm{E}) \operatorname{curl}_{\Gamma} \mathcal{G}_{T}+\lambda\left(2 R-2 H_{\Gamma}\right) \mathrm{H}_{T} \cdot \mathcal{G}\right. \\
& \left.+\lambda(\nu \cdot \mathrm{H}) \operatorname{div}_{\Gamma} \mathcal{G}_{T}+2 H_{\Gamma} \eta \operatorname{curl}_{\Gamma} \mathrm{H}_{T} \operatorname{curl}_{\Gamma} \mathcal{G}_{T}+i k \mathcal{Z} \mathrm{H}_{T} \cdot \mathcal{Z} \mathcal{G}_{T}\right\}
\end{aligned}
$$

We evaluate $\mathcal{Z} \mathrm{H}_{T}$ by first computing $H_{\eta}$, the $L^{2}$ projection of $\eta \operatorname{curl}_{\Gamma} \mathrm{H}_{T}$ on piecewise linear functions that solves

$$
\int_{\Gamma} H_{\eta} v d s=\int_{\Gamma}\left(\eta \operatorname{curl}_{\Gamma} \mathrm{H}_{T}\right) v d s
$$

for all $v$ in the space of piecewise linear functions defined on the triangular discretisation of $\Gamma$. We then compute $\mathcal{Z} \mathrm{H}_{T}$ by evaluating $\operatorname{curl}_{\Gamma} H_{\eta}$ as a piecewise constant function.

We numerically validate both, the characterization of the shape derivative and its numerical evaluation, by comparing expression (17) with a numerical evaluation of

$$
\partial_{\varepsilon} F(\Gamma):=\frac{F(\Gamma)-F\left(\Gamma_{t}\right)}{t},
$$

for $\Gamma_{t}:=\Gamma+t \varepsilon(\Gamma)$ where $t$ is a small positive number and $\varepsilon$ is a vector field defined on $\Gamma$. We also used finite elements to compute $\partial_{\varepsilon} F(\Gamma)$. 


\section{Numerical Results}

We will consider data $\mathrm{E}_{\mathrm{obs}}^{\infty}$ that correspond with the scattering of an incident plane wave of frequency $k=4$, of direction of direction of incidence $\hat{\theta}=(0,0,1)$ and of polarisation $\mathbf{p}=(1,0,0)$. Moreover we set $\eta=-\lambda=i / k$ and the obstacle $\Omega_{\mathrm{obs}}$ is a sphere of radius $0.3 l$ where $l:=2 \pi / k$ is the wavelength of the incident wave. The obstacle $\Omega$ is taken as being a sphere of radius $0.5 l$ and the deformation is $\varepsilon=\hat{x}=x /|x|$. In this case, $(20)$ can also be computed via Mie's series for $\varepsilon=\hat{x}$ (we denote by $\widetilde{\partial F}_{\varepsilon}$ this value) and we have the following results.

$$
\partial_{\varepsilon} F(\Gamma)=11.7, \quad \widetilde{\partial F}_{\varepsilon}(\Gamma)=11.3, \quad F^{\prime}(\Gamma)=10.9 .
$$

These three values are fairly close given that we have a precision of approximately $5 \%$ on the forward solver. Considering an artificial boundary $\Sigma$ as close as one wavelength from the scatterer is certainly not enough to achieve such precision for more complex objects such as the cube use below. Nevertheless, the accuracy we obtained is certainly good enough for illustration purposes.

\section{Application to shape identification of obstacles with GIBC}

We propose in this section to use the characterization of the shape derivative obtained previously to reconstruct the shape $\Gamma$ of a scatterer with boundary conditions of the form

$$
\nu \times \mathrm{E}+\operatorname{curl}_{\Gamma}\left(\eta \operatorname{curl}_{\Gamma} \mathrm{H}_{T}\right)+\lambda \mathrm{H}_{T}=0 \quad \operatorname{sur} \Gamma
$$

with constant $\lambda$ and $\eta$, from the knowledge of the far field produced by the scattering of $J$ incident plane waves. We first present the proposed regularised steepest descent minimization procedure and then show some numerical validating results.

\subsection{Reconstruction procedure}

The technique we used to solve the inverse problem consists in minimizing the following cost functional

$$
F_{J}(\Gamma):=\frac{1}{2} \sum_{j=1}^{J}\left\|T\left(\Gamma, \hat{\theta}_{j}, \mathbf{p}_{j}\right)-\mathrm{E}_{j, \delta}^{\infty}\right\|_{L_{t}^{2}\left(S^{2}\right)}^{2},
$$

where the far field patterns $\mathrm{E}_{j, \delta}^{\infty}$ represent the data and we do not mention the dependence of $T$ on $\lambda$ and $\eta$ for simplicity reasons. For more clarity we made explicit the dependence of $T$ on the direction of incidence $\hat{\theta_{j}}$ and the polarisation $\mathbf{p}_{j}$ of the incident plane wave. Therefore, we obtain the shape derivative of $F_{J}$ in the direction $\varepsilon$ as being given by

$$
F_{J}^{\prime}(\Gamma) \cdot \varepsilon:=\frac{1}{4 \pi} \sum_{j=1}^{J} \Re\left(\int_{\Gamma} \mathcal{G}_{j}(y) \cdot B_{\varepsilon}\left(\mathrm{E}_{j}, \mathrm{H}_{j}\right)(y) d s(y)\right),
$$

where $B_{\varepsilon}$ is given in Theorem 4.1, $\mathrm{E}_{j}(y)=\mathscr{E} s\left(y, \hat{\theta}_{j}\right) \mathbf{p}_{j}+\mathscr{E}^{i}\left(y, \hat{\theta}_{j}\right) \mathbf{p}_{j}, \mathrm{H}_{j}(y)=\mathrm{H}^{s}\left(y, \hat{\theta}_{j}\right) \mathbf{p}_{j}+\mathrm{H}^{i}\left(y, \hat{\theta}_{j}\right) \mathbf{p}_{j}$ and the adjoint state $\mathcal{G}_{j}$ is given by

$$
\mathcal{G}_{j}(y):=\int_{S^{2}} \mathscr{H}_{j}(y,-\hat{x}) \mathbf{g}_{j}(\hat{x}) d s(\hat{x})
$$

where $\mathbf{g}_{j}(\hat{x}):=\left(\overline{T\left(\Gamma, \hat{\theta}_{j}, \mathbf{p}_{j}\right)-\mathrm{E}_{j, \delta}^{\infty}}\right)(\hat{x})$. We assume moreover that the impedance coefficients $\lambda$ and $\eta$ are constant, fixed and known and that for $j=1, \cdots, J$, the far field patterns $\mathrm{E}_{j, \delta}^{\infty}$ are

$$
\mathrm{E}_{j, \delta}^{\infty}=T\left(\Gamma_{0}, \hat{\theta}_{j}, \mathbf{p}_{j}\right)+\delta_{j}
$$

for some surface $\Gamma_{0}$ and "small" functions $\delta_{j} \in L_{t}^{2}(\Gamma)$ (the noise). The case of known but non constant coefficients requires a careful treatment since the coefficients would be known and defined on a shape that is unknown. One could for example assume that the unknown object can be described by a function of two variable $X$ and $Y$ and define the coefficients as functions of $X$ and $Y$. Another solution would consist in defining the coefficients in $\mathbb{R}^{3}$ but their physical significance would not be completely clear anymore. For more details on non constant impedances we refer to [6] that considers a similar problem for the scaler Helmholtz equation. 


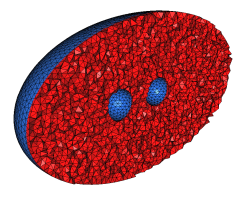

(a) Iteration 0

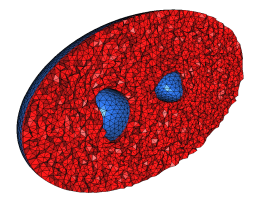

(b) Iteration 8

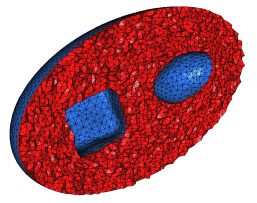

(c) Iteration 30

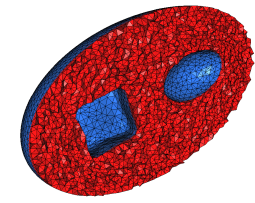

(d) Iteration 50

Figure 1: Different meshes obtained during the minimization procedure corresponding to Figure 4.

In the experiments hereafter we numerically compute an exact data set $T\left(\Gamma_{0}, \hat{\theta}_{j}, \mathbf{p}_{j}\right)$ by using finite elements and the $\delta_{i}$ are modeled by a realisation of a Gaussian random variable with mean 0 and variance 1. The $\delta_{j}$ are then normalised such that

$$
\frac{\left\|\delta_{j}\right\|_{L_{t}^{2}\left(S^{2}\right)}}{\left\|T\left(\Gamma_{0}, \hat{\theta}_{j}, \mathbf{p}_{j}\right)\right\|_{L_{t}^{2}\left(S^{2}\right)}}=\delta_{0},
$$

for a given noise level $\delta_{0}>0$.

We propose to use a steepest descent method to minimize $F_{J}$. At each step $n$ we update the current shape $\Gamma_{n}$ by moving it with a regular representation of the shape derivative of $F$. More precisely, we begin with a given initial mesh $M_{0}$ obtained from the discretisation of the domain contained between an initial guess for the geometry $\Gamma$ and the auxiliary boundary $\Sigma$. Then we move this mesh iteratively until convergence. For a given step $n$ of the algorithm we obtain the mesh at step $n+1$, which is denoted $M_{n+1}$, by applying the following deformation to the current mesh $M_{n}$ :

$$
M_{n+1}:=\left(\mathrm{Id}+\alpha_{n} V\right) M_{n},
$$

where $V \in X_{h}$ solves

$$
(V, W)_{\left(H^{1}\left(M_{n}\right)\right)^{3}}+\sigma_{\Gamma}\left(\nabla_{\Gamma} V, \nabla_{\Gamma} W\right)_{\left(L_{t}^{2}\left(\Gamma_{n}\right)\right)^{3 \times 3}}+(V, W)_{\left(L_{t}^{2}\left(\Gamma_{n}\right)\right)^{3}}=F_{J}^{\prime}\left(\Gamma_{n}\right) \cdot W,
$$

for all $W \in X_{h}$ where $X_{h}$ is the set of piecewise linear Lagrange finite elements defined on the mesh $M_{n}$ that vanish on the exterior boundary $\Sigma$ (see [2] for a similar approach). The parameter $\alpha_{n}>0$ is the step size, $\sigma_{\Gamma}>0$ is a regularisation coefficient and $F_{J}^{\prime}\left(\Gamma_{n}\right) \cdot v$ is given by formula (21). One advantage of this approach is that it is a parametrisation free procedure (see Figure 1 for an example of successive meshes obtained during the iterative process). The inverse crime is automatically avoided since the mesh is different at each step.

The regularisation parameter is kept fixed during iterations while the step size evolves in the following way: we initialise it with a relatively small value and increase it by a multiplicative parameter $r>1$ if the cost functional decreases between mesh $M_{n}$ and $M_{n+1}$. On the contrary, we decrease it by the same multiplicative parameter $r$ if the cost functional increases between meshes $M_{n}$ and $M_{n+1}$ and we move back to the mesh $M_{n}$. A direct consequence of such approach is that the algorithm does not diverge and we can choose to stop the iterations when the step size is too small. To keep reasonably short computations (a few hours on a twelve core workstation) we choose to stop the algorithm after a sufficiently large number of iterations. In the experiments hereafter we observed that 40 iterations is generally sufficient to obtain a shape which barely evolves between iterations. We observe for instance tiny differences between Figure 1(c) and Figure 1(d). Finally, let us mention that moving meshes in dimension three is certainly a complicated subject and we used the free software mmg3d (see [15]) to perform this step. We also used the mesh adaptation capabilities of mmg3d at every step to further ensure that the mesh stays regular enough after each iteration. We can summarise the algorithm as follows:

Initialisation: pick an initial mesh $M$, an initial step size $\alpha$, solve equations (18)-(19) for all incident plane waves and evaluate the initial cost functional $F_{J}$

Iterations: until $\alpha<\alpha_{\min }$ or the maximum number of iterations is reached 
1. compute the $I$ adjoint states $\mathcal{G}$ (one for each incident wave)

2. compute a descent direction $V$ by solving equation (22)

3. define a new mesh $\tilde{M}:=(\mathrm{Id}+\alpha V) M$

4. solve equations (18)-(19) in $\tilde{M}$ for all incident plane waves and evaluate the cost functional $\tilde{F}$

5. if $\tilde{F}<F$ else

$$
\alpha:=r \alpha, M:=\tilde{M}, F:=\tilde{F},
$$

$\alpha:=\alpha / r$ and go back to step 3.

\subsection{Numerical results}

We now present three examples of reconstructions obtained using the procedure described here above. Our goal is to show how the algorithm behaves with respect to the shape complexity and to demonstrate that our choice of minimization procedure (regularisation of the gradient and parametrisation free optimisation) gives valuable results. For each example we plot three shapes: the exact shape $\Gamma_{0}$ used to simulate the data, the initial guess and the shape obtained at the $40^{\text {th }}$ iteration of the minimization algorithm. The blue line represents the wavelength of the incident wave, in all cases we considered an obstacle which was about the size of the wavelength and we used the same parameters: the regularisation coefficient for the computation of the shape gradient is $\sigma_{\Gamma}=0.3$, the initial step size is $\alpha_{0}=0.1$, the actualisation rate for the step size is $r=1.2$ and the level of noise is $\delta_{0}=2 \%$. We recall that the incident plane waves that are used to generate the data are of the form

$$
\begin{aligned}
& \mathscr{E}^{i}(x, \hat{\theta}) \mathbf{p}:=-\frac{1}{i k} \operatorname{curl}_{x} \mathbf{c u r l}_{x}\left(\mathbf{p} e^{i k x \cdot \hat{\theta}}\right)=i k((\hat{\theta} \times \mathbf{p}) \times \hat{\theta}) e^{i k x \cdot \hat{\theta}}, \\
& \mathscr{H}^{i}(x, \hat{\theta}) \mathbf{p}:=\operatorname{curl}_{x}\left(\mathbf{p} e^{i k x \cdot \hat{\theta}}\right)=i k(\hat{\theta} \times \mathbf{p}) e^{i k x \cdot \hat{\theta}}
\end{aligned}
$$

and are characterised by their direction of incidence $\hat{\theta}$ and polarisation $\mathbf{p}$.

In the first test (Figure 2) we considered data from 2 incident waves while in the second and third one we considered four incident waves since the shape reconstruction is more challenging $\left(\Gamma_{0}\right.$ is non convex in Figure 3 or non connected in Figure 4). In the three cases the reconstructions are accurate and comparable to the one obtained with the so called Linear Sampling Method (see [13]) that requires the use of many more incident waves (but indeed is much less expensive and does not need a priori initial guesses).

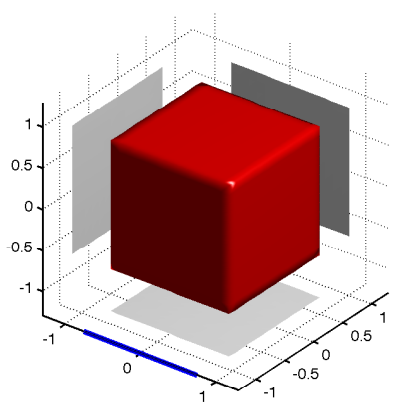

(a) Exact shape

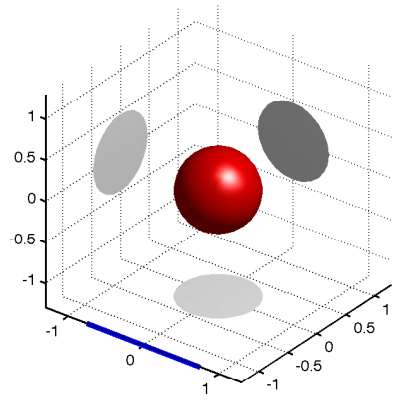

(b) Initial guess

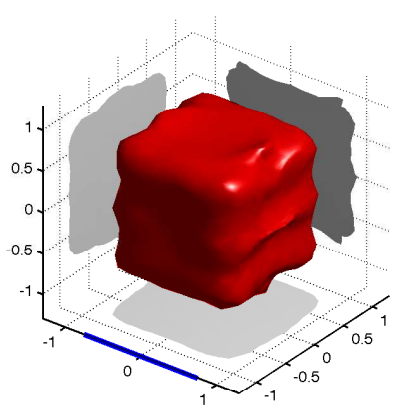

(c) Iteration 40

Figure 2: Reconstruction with $\delta_{0}=2 \%$ of noise and for two incident waves : $\hat{\theta}_{1}=-\hat{\theta}_{2}=(0,0,1)$ and $\mathbf{p}_{1}=\mathbf{p}_{2}=(0,1,0)$. The impedance parameters are $\lambda=0.5$ and $\eta=0$. 


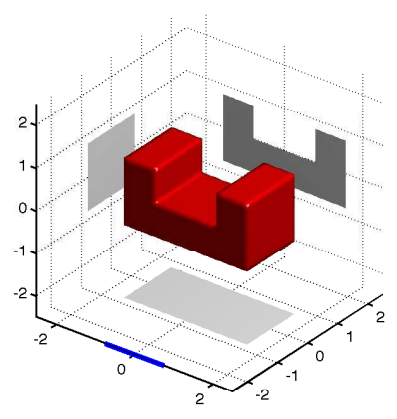

(a) Exact shape

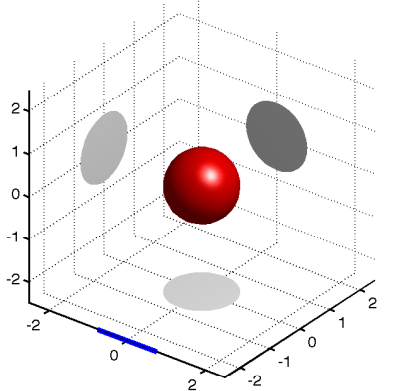

(b) Initial guess

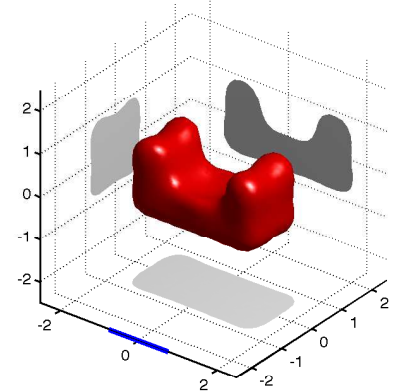

(c) Iteration 40

Figure 3: Reconstruction with $\delta_{0}=2 \%$ of noise and for four incident waves : $\hat{\theta}_{1}=-\hat{\theta}_{2}=(0,0,1)$, $\hat{\theta}_{3}=-\hat{\theta}_{4}=(0,1,0), \mathbf{p}_{1}=\mathbf{p}_{2}=(0,1,0)$ and $\mathbf{p}_{3}=\mathbf{p}_{4}=(1,0,0)$. The impedance parameters are $\lambda=-0.25 i$ and $\eta=0.25 i$.

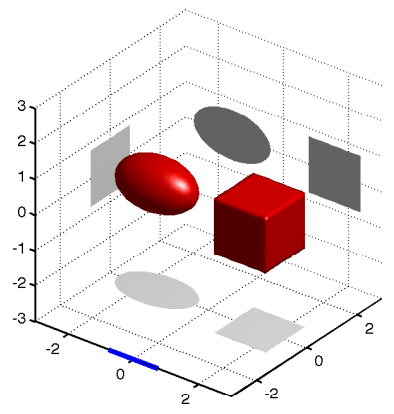

(a) Exact shape

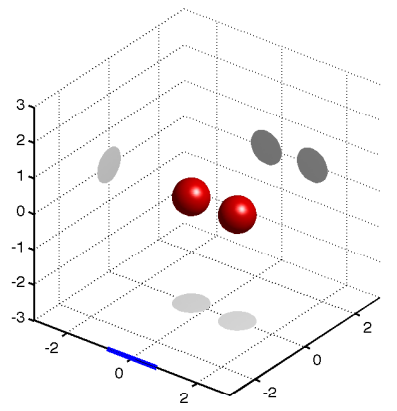

(b) Initial guess

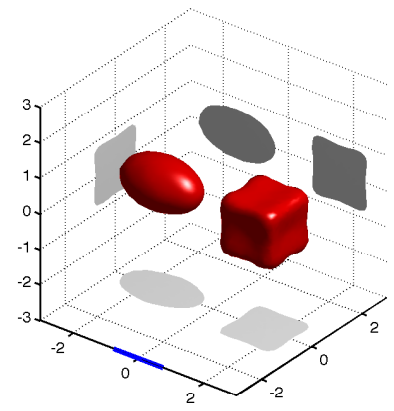

(c) Iteration 40

Figure 4: Reconstruction with $\delta_{0}=2 \%$ of noise and for four incident waves : $\hat{\theta}_{1}=-\hat{\theta}_{2}=(0,0,1)$, $\hat{\theta}_{3}=-\hat{\theta}_{4}=(0,1,0), \mathbf{p}_{1}=\mathbf{p}_{2}=(0,1,0)$ and $\mathbf{p}_{3}=\mathbf{p}_{4}=(1,0,0)$. The impedance parameters are $\lambda=-0.25 i$ and $\eta=0.25 i$. 


\section{Appendix}

Proof of Lemma 4.9. We use the notation introduced in Section 4.2. We complement the tangent basis $\left(e_{1}, e_{2}\right)$ (associated with the local parametrisation $\left.\varphi(14)\right)$ with $e_{3}=\varepsilon$. This defines a non orthogonal basis of $\mathbb{R}^{3}$ and we denote by $\left(f^{1}, f^{2}, f^{3}\right)$ the associated covariant basis. Let us also introduce $\left(e^{1}, e^{2}, e^{3}\right)$ the covariant basis associated with $\left(e_{1}, e_{2}, \nu\right)$. One easily verifies (see also [6]):

$$
f^{1}=e^{1}-\frac{1}{\nu \cdot \varepsilon}\left(e^{1} \cdot \varepsilon\right) \nu, f^{2}=e^{2}-\frac{1}{\nu \cdot \varepsilon}\left(e^{2} \cdot \varepsilon\right) \nu \quad \text { and } f^{3}=\frac{1}{\nu \cdot \varepsilon} \nu .
$$

We also denote by $\widetilde{\nu}_{t}$ the extension of the normal vector $\nu$ given by

$$
\widetilde{\nu}_{t}\left(x_{\Gamma}+t \nu\left(x_{\Gamma}\right)\right)=\nu\left(x_{\Gamma}\right) \quad \text { for } x_{\Gamma} \in \Gamma .
$$

Let us prove the first identity of Lemma 4.9. By using [28, Theorem 2.5.20] we have

$$
\left.\operatorname{curl}\left(\nu_{t} \times V\right)\right|_{t=0}=\operatorname{div}_{\Gamma}\left(V_{T}\right) \nu+\left.\left(R-2 H_{\Gamma} \operatorname{Id}-\frac{\partial}{\partial \nu}\right)\left[\left(\nu_{t} \times V\right) \times \widetilde{\nu}_{t}\right]\right|_{t=0}
$$

since

$$
\left.\operatorname{curl}_{\Gamma}\left(\left[\nu_{t} \times V\right] \cdot \widetilde{\nu}_{t}\right)\right|_{t=0}=\operatorname{curl}_{\Gamma}([\nu \times V] \cdot \nu)=0 .
$$

Moreover,

$$
\left.\frac{\partial}{\partial \nu}\left[\left(\nu_{t} \times V\right) \times \widetilde{\nu}_{t}\right]\right|_{t=0}=\left.\frac{\partial}{\partial \nu}\left[\left(\left(\nu_{t}-\widetilde{\nu}_{t}\right) \times V\right) \times \widetilde{\nu}_{t}\right]\right|_{t=0}+\frac{\partial}{\partial \nu} V_{T}
$$

For any vector field $W$,

$$
\left.\frac{\partial W}{\partial \nu}\right|_{\Gamma}=\sum_{i=1}^{2} \frac{\partial W}{\partial \xi_{i}}\left(f^{i} \cdot \nu\right)+\frac{\partial W}{\partial t}\left(f^{3} \cdot \nu\right)
$$

Hence we end up with

$$
\left.\frac{\partial}{\partial \nu}\left[\left(\left(\nu_{t}-\widetilde{\nu}_{t}\right) \times V\right) \times \widetilde{\nu}_{t}\right]\right|_{t=0}=\left[\left.\frac{\partial}{\partial t}\left(\nu_{t}-\widetilde{\nu}_{t}\right)\right|_{t=0} \times V\right] \times \nu=-\left[\nabla_{\Gamma}(\nu \cdot \varepsilon) \times V\right] \times \frac{\nu}{\nu \cdot \varepsilon}
$$

where for the first equality we used that for $i=1,2$

$$
\left.\frac{\partial}{\partial \xi_{i}}\left[\left(\left(\nu_{t}-\widetilde{\nu}_{t}\right) \times V\right) \times \widetilde{\nu}_{t}\right]\right|_{t=0}=0
$$

and for the second equality we used [18, Lemma 2.3]. This gives the first identity of Lemma 4.9.

For the second identity of Lemma 4.9 we first remark that

$$
\left.\frac{\partial}{\partial \nu}\left(\operatorname{curl}_{\Gamma_{t}} V_{T_{t}}\right)\right|_{t=0}=\left.\frac{\partial}{\partial \nu}\left(\nu_{t} \cdot \operatorname{curl} V\right)\right|_{t=0}=\left.\frac{\partial}{\partial \nu}\left(\widetilde{\nu}_{t} \cdot \operatorname{curl} V\right)\right|_{t=0}+\left.\frac{\partial}{\partial \nu}\left[\left(\nu_{t}-\widetilde{\nu}_{t}\right) \cdot \operatorname{curl} V\right]\right|_{t=0}
$$

Similarly we prove that

$$
\left.\frac{\partial}{\partial \nu}\left[\left(\nu_{t}-\widetilde{\nu}_{t}\right) \cdot \operatorname{curl} V\right]\right|_{t=0}=-\left.\frac{1}{\nu \cdot \varepsilon} \nabla_{\Gamma}(\nu \cdot \varepsilon) \cdot(\operatorname{curl} V)\right|_{\Gamma}
$$

We denote

$$
\operatorname{rot}_{\widetilde{\Gamma}_{t}}\left(\left(\widetilde{\nu}_{t} \times V\right) \times \widetilde{\nu}_{t}\right):=\widetilde{\nu}_{t} \cdot \operatorname{curl} V
$$

and

$$
\operatorname{div}_{\widetilde{\Gamma}_{t}}\left(V \times \widetilde{\nu}_{t}\right):=\operatorname{rot}_{\widetilde{\Gamma}_{t}}\left(\left(\widetilde{\nu}_{t} \times V\right) \times \widetilde{\nu}_{t}\right) .
$$

From [28, Lemma 2.5.10] we have

$$
\left.\frac{\partial}{\partial \nu}\left[\operatorname{div}_{\widetilde{\Gamma}_{t}}\left(V \times \widetilde{\nu}_{t}\right)\right]\right|_{t=0}-\left.\operatorname{div}_{\widetilde{\Gamma}_{t}}\left[\frac{\partial}{\partial \nu}\left(V \times \widetilde{\nu}_{t}\right)\right]\right|_{t=0}=-\operatorname{div}_{\Gamma}(R V)+2\left[(V \times \nu) \cdot \nabla_{\Gamma} H_{\Gamma}\right] .
$$

We also observe that

$$
\begin{aligned}
\left.\operatorname{div}_{\widetilde{\Gamma}_{t}}\left[\frac{\partial}{\partial \nu}\left(V \times \widetilde{\nu}_{t}\right)\right]\right|_{t=0} & =\operatorname{div}_{\Gamma}\left[\frac{\partial}{\partial \nu}(V \times \nu)\right] \\
& =\operatorname{div}_{\Gamma}\left[-\operatorname{curl} V+\left(\operatorname{curl}_{\Gamma} V_{T}\right) \nu+\left(R-2 H_{\Gamma}\right)(V \times \nu)\right]
\end{aligned}
$$


and

$$
-(\operatorname{curl} V) \cdot \nu+\operatorname{curl}_{\Gamma} V_{T}=0 .
$$

Whence

$$
\left.\operatorname{div}_{\widetilde{\Gamma}_{t}}\left[\frac{\partial}{\partial \nu}\left(V \times \widetilde{\nu}_{t}\right)\right]\right|_{t=0}=\operatorname{div}_{\Gamma}\left[-(\operatorname{curl} V)_{T}+\left(R-2 H_{\Gamma}\right)(V \times \nu)\right] .
$$

Combining this last relation together with (25) gives

$$
\left.\frac{\partial}{\partial \nu}\left(\widetilde{\nu}_{t} \cdot \operatorname{curl} V\right)\right|_{t=0}=\left.\frac{\partial}{\partial \nu}\left[\operatorname{div}_{\widetilde{\Gamma}_{t}}\left(V \times \widetilde{\nu}_{t}\right)\right]\right|_{t=0}=-\operatorname{div}_{\Gamma}(\operatorname{curl} V)_{T}-2 H_{\Gamma} \operatorname{div}_{\Gamma}(V \times \nu) .
$$

Finally, we can conclude by plugging this last identity and (24) into (23).

\section{References}

[1] G. Allaire. Conception optimale de structures. Springer-Verlag, 2007.

[2] G. Allaire and O. Pantz. Structural optimization with FreeFem++. Structural and Multidisciplinary Optimization, 32(3):173-181, 2006.

[3] C. Amrouche, C. Bernardi, M. Dauge, and V. Girault. Vector potentials in three-dimensional nonsmooth domains. Math. Methods Appl. Sci., 21(9):823-864, 1998.

[4] A. Bendali and K. Lemrabet. Asymptotic analysis of the scattering of a time-harmonic electromagnetic wave by a perfectly conducting metal coated with a thin dielectric shell . Asymptotic Analysis, $57(3-4): 199-227,2008$.

[5] L. Bourgeois, N. Chaulet, and H. Haddar. Stable reconstruction of generalized impedance boundary conditions. Inverse Problems, 27(9):095002, 26, 2011.

[6] L. Bourgeois, N. Chaulet, and H. Haddar. On simultaneous identification of the shape and generalized impedance boundary condition in obstacle scattering. SIAM J. Sci. Comput., 34(3):A1824-A1848, 2012.

[7] L. Bourgeois and H. Haddar. Identification of generalized impedance boundary conditions in inverse scattering problems. Inverse Problems and Imaging, 4(1):19-38, 2010.

[8] F. Cakoni, D. Colton, and P. Monk. The linear sampling method in inverse electromagnetic scattering. CBMS-NSF Regional Conference Series in Applied Mathematics. SIAM, 2011.

[9] F. Cakoni and R. Kress. Integral equation methods for the inverse obstacle problem with generalized impedance boundary condition. Inverse Problems, 29:015005, 2013.

[10] M. Chamaillard, N. Chaulet, and H. Haddar. Analysis of the factorization method for a general class of boundary conditions. Inverse and Ill Posed Problems, 2013.

[11] N. Chaulet. Electromagnetic scattering problems with generalized impedance boundary conditions. arXiv:1312.1089.

[12] N. Chaulet. Modèles d'impédance généralisée en diffraction inverse. PhD thesis, École Doctorale de l'École Polytechnique, 2012.

[13] D. Colton, H. Haddar, and P. Monk. The linear sampling method for solving the electromagnetic inverse scattering problem. SIAM J. Sci. Comput., 24(3):719-731, 2002.

[14] D. Colton and R. Kress. Inverse acoustic and electromagnetic scattering theory, volume 93 of Applied Mathematical Sciences. Springer, New York, third edition, 2013.

[15] C. Dobrzynski. Mmg3d : Userguide. Technical report 442, INRIA, 2012.

[16] M. Duruflé, H. Haddar, and P. Joly. Higher order generalized impedance boundary conditions in electromagnetic scattering problems. C.R. Physique, 7(5):533-542, 2006. 
[17] H. Haddar, P. Joly, and H.-M. Nguyen. Generelized impedance boundary conditions for scattering problems from strongly absorbong obstacles: the case of Maxwell's equations. Math. Models Methods Appl. Sci., 18(10):1787-1827, 2008.

[18] H. Haddar and R. Kress. On the Fréchet derivative for obstacle scattering with an impedance boundary condition. SIAM J. Appl. Math., 65(1):194-208 (electronic), 2004.

[19] H. Harbrecht and T. Hohage. Fast methods for three-dimensional inverse obstacle scattering problems. J. Integral Equations Appl., 19(3):237-260, 2007.

[20] F. Hecht. New development in FreeFem++. J. Numer. Math., 20(3-4):251-265, 2012.

[21] T. Hohage. Fast numerical solution of the electromagnetic medium scattering problem and applications to the inverse problem. J. Comput. Phys., 214(1):224-238, 2006.

[22] O. Ivanyshyn, R. Kress, and P. Serranho. Huygens' principle and iterative methods in inverse obstacle scattering. Adv. Comput. Math., 33(4):413-429, 2010.

[23] A. Kirsch and R. Kress. Uniqueness in inverse obstacle scattering (acoustics). Inverse Problems, 9(2):285-299, 1993.

[24] R. Kress. Electromagnetic waves scattering: specific theoretical tools. In E. R. Pike and P. C. Sabatier, editors, Scattering, pages 175-190. Academic press, 2002.

[25] R. Kress and L. Päivärinta. On the far field in obstacle scattering. SIAM J. Appl. Math., 59(4):14131426 (electronic), 1999.

[26] R. Kress and W. Rundell. A quasi-Newton method in inverse obstacle scattering. Inverse Problems, 10(5):1145-1157, 1994.

[27] J.-C. Nédélec. Mixed finite elements in $\mathbb{R}^{3}$. Numer. Math., 35:315-341, 1980.

[28] J.-C. Nédélec. Acoustic and electromagnetic equations, volume 144 of Applied Mathematical Sciences. Springer-Verlag, New York, 2001. Integral representations for harmonic problems.

[29] R. Potthast. Point sources and multipoles in inverse scattering theory, volume 427 of Chapman 8 Hall/CRC Research Notes in Mathematics. Chapman \& Hall/CRC, Boca Raton, FL, 2001.

[30] M. Willatzen. Electromagnetic-wave propagation along curved surfaces. Phys. Rev. A, 80:043805, Oct 2009. 